TRANSACTIONS OF THE

AMERICAN MATHEMATICAL SOCIETY

Volume 349, Number 11, November 1997, Pages 4501-4535

S 0002-9947(97)02047-3

\title{
NONSYMMETRIC SYSTEMS ON NONSMOOTH PLANAR DOMAINS
}

\author{
G. C. VERCHOTA AND A. L. VOGEL
}

\begin{abstract}
We study boundary value problems, in the sense of Dahlberg, for second order constant coefficient strongly elliptic systems. In this class are systems without a variational formulation, viz. the nonsymmetric systems. Various similarities and differences between this subclass and the symmetrizable systems are examined in nonsmooth domains.
\end{abstract}

\section{INTRODUCTION}

After B. E. J. Dahlberg proved the mutual absolute continuity of harmonic measure with respect to surface (Lebesgue) measure on the boundary of Lipschitz domains [Dah77] and Coifman-McIntosh-Meyer [CMM82] completed the singular integral theory of [Cal77] on Lipschitz curves, one of the projects that received some considerable attention was that of establishing related dilation invariant estimates for real constant coefficient operators, including systems, other than the Laplacian. Recently the first author and J. Pipher [PV95a] showed, in particular, that such estimates are valid for the Dirichlet problem for the single homogeneous equation $L u=0$ with data in $L^{2}$ with respect to surface measure $d \sigma$ of the boundary $\partial \Omega$ of a Lipschitz domain $\Omega$ in $n$-dimensional Euclidean space. There $L$ could be of any order with the only conditions imposed being that $L$ be elliptic and consist of only a principal part. In [Ver96] it was shown that this could be extended to systems of equations $L u=\sum_{l=1}^{N} L^{k l} u_{l}=0, k=1, \ldots, N$, of all orders with ellipticity defined in the sense of Legendre-Hadamard and the only new condition imposed on $L$ being that of symmetry,

$$
L^{k l}=L^{l k} .
$$

Any weaker ellipticity than Legendre-Hadamard leads to nonuniqueness of solutions even in smooth domains (see §2), and nonsymmetry plays no role for constant coefficient single equations. The question then arises as to the necessity of symmetry conditions for elliptic systems when attempting to solve boundary value problems in the sense of Dahlberg, i.e. in the sense of nontangential limits a.e. $(d \sigma)$ on the boundaries of Lipschitz domains. We find some answers to this question in this

Received by the editors November 10, 1995.

1991 Mathematics Subject Classification. Primary 35J55, 31A25.

Key words and phrases. Elliptic, bianalytic, weak maximum principle, Rellich identity, boundary value problems, nonvariational.

The first author was partially supported by NSF Grant DMS-9401354. 
paper by considering the case of real second order Legendre-Hadamard systems of two equations in two unknowns in plane domains.

The most striking distinction between symmetric and nonsymmetric systems is that the $L^{2}(\partial \Omega)$ Dirichlet problem, which is always solvable in the symmetric case, fails to be solvable for some nonsymmetric systems in domains with just a single corner. Furthermore, the corner can be convex (rather than reentrant as with $p<2$ counterexamples for Laplace's equation [Dah77]) and have small Lipschitz constant. Also, failure of the solvability of the Dirichlet problem with $L^{p}(\partial \Omega)$ data can be demonstrated for any $p<\infty$. All of this follows by choosing nonsymmetric systems with canonical ellipticity $0<k<1$ (see $\S 2$ ) away from that for the Laplacian $k=1$. This may be demonstrated as in $\S 8$ by utilizing examples originally due to Kozlov on the spectrum of certain operator pencils in plane corners [Koz90]. By restricting attention to the nonsymmetric systems that are skewsymmetric (see $\S 2$ ) we also find in $\S 8$ that for these systems there is an interval of canonical ellipticity $0<k_{0}<k \leq 1$ for which no $L^{2}(\partial \Omega)$ counterexamples exist in domains with a finite number of corners. This follows variously from [CD93], which demonstrates that all singular solutions in corners must be those of $\S 8$, or from the positive results of $\S 6$ below on $C^{1}$ polygons. Thus there is a collection of nonsymmetric LegendreHadamard systems for which, as of this writing, there are for the class of Lipschitz domains neither counterexamples to nor proof of $L^{2}(\partial \Omega)$ solvability.

The failure to obtain positive $L^{2}(\partial \Omega)$ results in Lipschitz domains for this collection is a result of the nonavailability of certain boundary energy estimates that have become known in recent years as Rellich formulas. These formulas are available in any Lipschitz domain for any of the symmetrizable systems considered here (see [PV95a], [Ver96] and Remark 8 in $\S 6$ below).

As a consequence of the lack of dilation invariant energy estimates in some cases and $L^{p}(\partial \Omega), p \geq 2$, counterexamples in some others, we study in $\S 6$ the Dirichlet problem in $C^{1}$ polygons with data in $L^{p}(\partial \Omega), p<\infty$ large enough. As in [DL92], where the symmetrizable and triangular (§2) Legendre-Hadamard systems are studied, we use the analysis of the spectrum of the double layer potentials in $L^{p}(\partial \Omega), 1<p<\infty$, given in [LP80] and [LP83]. This is carried out in $\S 5$, to account for the rotational dependence of the spectrum, which ends with the observation in Lemma 5.1 that the double layer potential is Fredholm with zero index for any $p$ large enough, depending on the system under consideration. We also observe that the spectral analysis of $\S 5$ and the exponents on the power solutions which provide the counterexamples of $\S 8$ come from precisely the same computations (see (40) and (64)).

In $\S 6$, given any one of our systems, we demonstrate the solutions to three boundary value problems on $C^{1}$ polygons, viz. the Dirichlet problem for $p<\infty$ large enough and the Neumann and regularity problems for $p>1$ small enough. These results are then used in $\S 7$ to obtain the $L^{1}(\partial \Omega)$ estimate on the nontangential maximal function for solutions to the Neumann and regularity problems with data in the atomic Hardy space $H^{1}(\partial \Omega)$. This then leads as in [DK90] and [PV93] to the weak maximum principle on $C^{1}$ polygons for the Dirichlet problem. We carry out this project in detail because there are no dilation invariant estimates for solutions available for use when dealing with nonsymmetric systems while attempting to prove Hardy space estimates. This is in contrast to, for example, [FK81] or the $C^{1}$ domain estimates of [PV93], where in the background is the $L^{2}(\partial \Omega)$ dilation invariant theory of [Dah79] or [DKV86]. 
Solutions to the systems studied here are also known in the literature as bianalytic functions. The monograph [KWCQ85], which we found through [DL92], contains a great many useful facts about these functions. Particularly helpful is the notion of canonical form, which describes all elliptic systems (7) with just two parameters, clarifying the interaction between ellipticity and symmetry. See also [BG93].

We end the introduction with two open problems. First, since the weak maximum principle holds on $C^{1}$ polygons for any strongly elliptic system while for any $p<\infty$ a strongly elliptic system can be found for which the $L^{p}(\partial \Omega)$ Dirichlet problem is not solvable, is it possible that on general Lipschitz domains the $L^{\infty}(\partial \Omega)$ Dirichlet problem will always be uniquely solvable, i.e., given any strongly elliptic system and bounded Dirichlet data, will a unique bounded solution exist that takes on the data? Second, again confining our attention to the class of skewsymmetric systems $0<k<1$, we find below that for a given $k$ solutions are necessarily of the form $f((1+k) z-(1-k) \bar{z})+\overline{g(z)}=f \circ A(z)+\overline{g(z)}$, where $g$ is holomorphic on $\Omega, g\left(\zeta_{0}\right)=0$ for some fixed $\zeta_{0} \in \Omega$ and $f$ is holomorphic on the image of $\Omega$ under the linear transformation $A(z)=(1+k) z-(1-k) \bar{z}$ (see (50)). Therefore, we would have counterexamples to unique solvability of the Dirichlet problem in $L^{2}$ when there is a nonzero holomorphic function $f$ and a holomorphic function $g, g\left(\zeta_{0}\right)=0$, each with $L^{2}$ boundary values, such that $f \circ A=-\bar{g}$ on $\partial \Omega$. More generally, letting $\mathcal{F}=\left\{f \circ A \mid f \in H(A(\Omega))\right.$ and $\left.\|f \circ A\|_{L^{2}(\partial \Omega)} \leq 1\right\}$ and $\mathcal{G}=\left\{g \mid \bar{g} \in H(\Omega), g\left(\zeta_{0}\right)=0\right.$ and $\left.\|g\|_{L^{2}(\partial \Omega)} \leq 1\right\}$, we may ask, in the case when $\Omega$ is a Lipschitz domain, are the $L^{2}(\partial \Omega)$ closures of $\mathcal{F}$ and $\mathcal{G}$ at a positive angle, i.e. $\sup _{f \circ A \in \mathcal{F}, g \in \mathcal{G}}\left|\int_{\partial \Omega} f \circ A \bar{g} d \sigma\right|<1$ ? When $k=1$ the answer, provided by the HelsonSzegö Theorem (see [Gar81] and [Ken80, p. 135]), is yes. When $0<k \leq k_{0}$ the counterexamples of $\S 8$ show that the answer is no even in domains with just a single corner. But is there some Helson-Szegö type result when $k_{0}<k<1$ ?

Acknowledgement. The authors would like to thank Jill Pipher for telling us about her recent work with Carlos Kenig on the single divergence form elliptic equation with nonsymmetric coefficient matrix. It was this that led us to consider the consequences of nonsymmetry for the systems presented here.

\section{The SYstems, ELlipticity, AND SYMMetry}

Let $A, B$, and $C$ be real, constant $2 \times 2$ matrices. The general system we are concerned with has the form

$$
\left(A \frac{\partial^{2}}{\partial x^{2}}+2 B \frac{\partial^{2}}{\partial x \partial y}+C \frac{\partial^{2}}{\partial y^{2}}\right)\left(\begin{array}{l}
u(x, y) \\
v(x, y)
\end{array}\right)=\left(\begin{array}{l}
0 \\
0
\end{array}\right) .
$$

Following [KWCQ85], the characteristic matrix of this system is

$$
A \xi_{1}^{2}+2 B \xi_{1} \xi_{2}+C \xi_{2}^{2}
$$

The biquadratic characteristic form of the system is the determinant of the characteristic matrix,

$$
F(\vec{\xi})=\operatorname{det}\left(A \xi_{1}^{2}+2 B \xi_{1} \xi_{2}+C \xi_{2}^{2}\right) .
$$

The system is said to be elliptic if the biquadratic characteristic form is not zero for all $\vec{\xi}=\left(\begin{array}{l}\xi_{1} \\ \xi_{2}\end{array}\right) \in \mathbb{R}^{2} \backslash\{\overrightarrow{0}\}$. This is the standard definition of ellipticity [ADN64]. When $A=C=I$ and $B=0$ system (1) reduces to the two equations $\Delta u=0$ and 
$\Delta v=0$, and the biquadratic characteristic form is $F(\vec{\xi})=\left(\xi_{1}^{2}+\xi_{2}^{2}\right)^{2}$. System (1) may also be presented in the form

$$
\frac{\partial}{\partial x_{i}}\left(a_{i j}^{\alpha \beta} \frac{\partial u^{\beta}}{\partial x_{j}}\right)=0 \text { for } \alpha=1,2,
$$

where $u=u^{1}, v=u^{2}$ and $i, j, \beta$ are summed from 1 to 2 . Here $A_{\alpha \beta}=a_{11}^{\alpha \beta}$, $B_{\alpha \beta}=a_{12}^{\alpha \beta}=a_{21}^{\alpha \beta}$ and $C_{\alpha \beta}=a_{22}^{\alpha \beta}$ for $\alpha, \beta=1,2$. The ellipticity of system (4),

$$
\operatorname{det}\left(\begin{array}{cc}
\vec{\xi}^{T} a^{11} \vec{\xi} & \vec{\xi}^{T} a^{12} \vec{\xi} \\
\vec{\xi}^{T} a^{21} \vec{\xi} & \vec{\xi}^{T} a^{22} \vec{\xi}
\end{array}\right) \neq 0 \text { for } \vec{\xi} \in \mathbb{R}^{2} \backslash\{(0,0)\},
$$

is the same as the ellipticity of system (1) defined above.

We will use three types of transformations on the systems (1): (i) nonsingular linear combination of equations, (ii) nonsingular linear combination of dependent variables, and (iii) nonsingular linear combination of independent variables. Transformations of types (i) and (ii) are achieved by multiplying $A, B$, and $C$ in system (1) on the left and right respectively by a nonsingular $2 \times 2$ matrix. If after performing a finite number of these transformations at least one of the equations is uncoupled, the system is said to be reducible. Solving such a system amounts to solving two equations successively, so we will generally consider nonreducible systems.

Using one coordinate transformation (type (iii)), every nonreducible elliptic system (1) may be transformed [KWCQ85, Chapter 1, section 3] into a system whose biquadratic characteristic form is

$$
\left(\xi_{1}^{2}+\xi_{2}^{2}\right)\left(\xi_{1}^{2}+k^{2} \xi_{2}^{2}\right) \text { with } 0<k \leq 1 .
$$

We will call $k$ the canonical ellipticity of the system. The idea behind the transformation is to find a real linear fractional transformation in the complex plane that sends the complex conjugate pairs of roots of the system's biquadratic characteristic form to $\pm i, \pm i k$. By applying several more transformations, all of type (i) or (ii), any nonreducible elliptic system may put in the canonical form

$$
\left(\left(\begin{array}{cc}
1 & 0 \\
0 & \frac{\lambda}{k^{2}}
\end{array}\right) \frac{\partial^{2}}{\partial x^{2}}+\left(\begin{array}{cc}
0 & \frac{\lambda-k^{2}}{k} \\
\frac{\lambda-1}{k} & 0
\end{array}\right) \frac{\partial^{2}}{\partial x \partial y}+\left(\begin{array}{cc}
\lambda & 0 \\
0 & 1
\end{array}\right) \frac{\partial^{2}}{\partial y^{2}}\right)\left(\begin{array}{l}
u \\
v
\end{array}\right)=\left(\begin{array}{l}
0 \\
0
\end{array}\right)
$$

where $\lambda \neq 0,1, k^{2}$ and $0<k \leq 1$ [KWCQ85, Chapter 1 , section 4]. Note that when $\lambda=1$ or $k^{2}$ the system is reducible. We shall also call a system (7) with $\lambda=1$ or $k^{2}$ triangular.

It is easily checked that all elliptic systems (7) satisfy the supplementary condition in [ADN64]. Further, except for $\lambda=-k$ they also satisfy the complementing boundary condition (Lopatinskiî-Šapiro condition) in [ADN64]. Bitsadze [Bit48] has an example for the case $\lambda=-k$ showing the nonuniqueness of solutions to the Dirichlet problem in a ball. Let $A=\left(\begin{array}{ll}1 & 0 \\ 0 & 1\end{array}\right), B=\left(\begin{array}{cc}0 & -1 \\ 1 & 0\end{array}\right)$, and $C=\left(\begin{array}{cc}-1 & 0 \\ 0 & -1\end{array}\right)$. With these choices system (1) is elliptic. Now $u=v=1-x^{2}-y^{2}$ solves system (1) in the unit ball with zero boundary values. Furthermore, the estimates up to the boundary of [ADN64] must fail for such systems.

Remark 1. For $\lambda<0$ but $\lambda \neq-k$ the systems (7) are elliptic and do satisfy the complementing boundary condition, so that the estimates of [ADN64] are valid in smooth domains. Nevertheless, there are polynomial examples of nonuniqueness 
for the Dirichlet problem in ellipses for such systems [KWCQ85, Chapter 1, section $7]$.

To overcome these difficulties [KWCQ85] introduce the notion of strong ellipticity due to $\left[\mathrm{SK}^{+} 60\right]$. System (1) is strongly elliptic if

$$
\operatorname{det}(A+2 \beta B+\gamma C) \neq 0 \text { for all } \beta^{2} \leq \gamma .
$$

Strong ellipticity is necessary and sufficient for the uniqueness of classical solutions to the Dirichlet problem for system (1) in arbitrary bounded smooth domains in the plane [KWCQ85, Chapter 1, section 7]. The question then arises as to how strong ellipticity compares to the Legendre-Hadamard condition for system (1) or (4), viz.

$$
a_{i j}^{\alpha \beta} \eta^{\alpha} \xi_{i} \eta^{\beta} \xi_{j}=\vec{\xi}^{T}\left(\begin{array}{ll}
\vec{\eta}^{T} A \vec{\eta} & \vec{\eta}^{T} B \vec{\eta} \\
\vec{\eta}^{T} B \vec{\eta} & \vec{\eta}^{T} C \vec{\eta}
\end{array}\right) \vec{\xi} \geq c|\vec{\eta}|^{2}|\vec{\xi}|^{2}
$$

for all $\vec{\eta}, \vec{\xi} \in \mathbb{R}^{2}$ and some positive constant $c$. [KWCQ85] shows that

Proposition 1. The condition of strong ellipticity is equivalent to the existence of transformations (of types (i) and (ii) above) that allow the system to be put in a form satisfying the Legendre-Hadamard condition.

Remark 2. [KWCQ85] also shows that (7) is strongly elliptic if and only if $\lambda>0$. That (7) is not strongly elliptic for $\lambda<0$ is easily seen by choosing $\beta=0$ and $\gamma=-1 / \lambda$ or $-\lambda / k^{2}$. We note that strong ellipticity is invariant under all three types of transformations listed above, while the Legendre-Hadamard condition is not invariant under transformations of types (i) and (ii).

To see that every strongly elliptic system may be transformed into a system which satisfies the Legendre-Hadamard condition (9), simply multiply the canonical form (7) on the left by the matrix

$$
\left(\begin{array}{cc}
\left|\frac{k}{\lambda-k^{2}}\right| & 0 \\
0 & \left|\frac{k}{\lambda-1}\right|
\end{array}\right)
$$

and calculate (9). The effect of this transformation is to make the coefficient matrix of the mixed partial derivatives in $(7)$ either $\left(\begin{array}{cc}0 & \pm 1 \\ \pm 1 & 0\end{array}\right)$ or $\left(\begin{array}{cc}0 & \pm 1 \\ \mp 1 & 0\end{array}\right)$. The former case is obtained when $\lambda>1$ or $\lambda<k^{2}$ and the system has been transformed into a symmetric Legendre-Hadamard system (see (4)). These systems are well understood [DKV88], [DK90] [DL92], [Ver96]. In the latter case, $k^{2}<\lambda<1$ and we are left with a system which is skewsymmetric. These are the nonsymmetric systems that are the focus of this paper. On the boundary between these two types of systems, $\lambda=1$ or $k^{2}$, lie the triangular systems. We will call the systems with $\lambda=k$ the skewsymmetric systems.

Remark 3. Using the coordinate transformation $\left(\begin{array}{ll}0 & 1 \\ k & 0\end{array}\right)$, system (7) generates a new canonical form with $\tilde{\lambda}=k^{2} / \lambda$ replacing $\lambda$ in (7). This transformation reflects the values of $\lambda>0$ in the point $k$ to obtain the values of $\tilde{\lambda}$. For example, the values of $\lambda$ in $\left(0, k^{2}\right)$ are reflected to the values of $\tilde{\lambda}$ in $(1, \infty)$. Thus in studying strongly elliptic systems it suffices to consider $\lambda \geq k$. The parameter $\lambda$ may be considered a measure of symmetry. 
It will be convenient to work with the system in yet a different form, in which many of the algebraic manipulations of matrices are reduced to multiplication of complex numbers. Multiplying the second equation in system (7) by $k$, setting $w=$ $u+i v$, and using the complex derivatives $\partial=\frac{1}{2}\left(\frac{\partial}{\partial x}-i \frac{\partial}{\partial y}\right)$ and $\bar{\partial}=\frac{1}{2}\left(\frac{\partial}{\partial x}+i \frac{\partial}{\partial y}\right)$, we obtain the single complex equation

$$
\partial M(\lambda, k) \bar{\partial}_{k} w=0,
$$

where $M(\lambda, k)=\left(1+\frac{\lambda}{k}\right)+\left(1-\frac{\lambda}{k}\right) \mathcal{C}, \bar{\partial}_{k}=(1+k) \bar{\partial}+(1-k) \partial$, and $\mathcal{C}$ is the conjugation operator. We denote the operator in (10) by $L=L(\lambda, k)=\partial M(\lambda, k) \bar{\partial}_{k}=\partial M \bar{\partial}_{k}$. Setting $\widetilde{M}=\widetilde{M}(\lambda, k)=\left(1+\frac{\lambda}{k}\right)-\left(1-\frac{\lambda}{k}\right) \mathcal{C}$, we also write $(10)$ with the derivatives gathered as

$$
\left(\partial \bar{\partial}(M+k \widetilde{M})+\partial^{2}(M-k \widetilde{M})\right) w=0 .
$$

Remark 4 (The operators $M$ ). For all complex numbers $a, b$ the operator $a+b \mathcal{C}$ is defined by $(a+b \mathcal{C})(w)=a w+b \bar{w}$. It has norm $|a|+|b|$ as an operator from $\mathbb{C}$ to $\mathbb{C}$ and inverse $\frac{\bar{a}-b \mathcal{C}}{|a|^{2}-|b|^{2}}$ as long as its determinant $|a|^{2}-|b|^{2} \neq 0$. This notation is just a convenient method of writing real $2 \times 2$ matrices. Let $w=u+i v$, $a=x+i y$ and $b=r+i s$ with $u, v, x, y, r, s$ real. Then the result $(a+b \mathcal{C})(w)$, thought of as vector in $\mathbb{R}^{2}$, is the same as $\left(\begin{array}{cc}x+r & -y+s \\ y+s & x-r\end{array}\right)\left(\begin{array}{l}u \\ v\end{array}\right)$. With this in mind it is easy to see that the transpose may be defined and is given by $(a+b \mathcal{C})^{T}=\bar{a}+b \mathcal{C}$. The conjugate transpose $\overline{a+b \mathcal{C}}^{T}=a+\bar{b} \mathcal{C}$ arises naturally in expressions such as $\Re[z(a+b \mathcal{C}) w]=\Re[w(a+\bar{b} \mathcal{C}) z]$, where $z, w$ are any complex numbers and $\Re$ denotes the real part operator. We shouldn't but we will call the operator Hermitian if and only if $b$ is real, since then $\overline{a+b \mathcal{C}}^{T}=a+b \mathcal{C}$. Further, thinking of complex numbers as representing column vectors, the first column of $a+b \mathcal{C}$ is $a+b$ and the second is $i(a-b)$. Sometimes we will write $a^{-1} \mathcal{C}=\mathcal{C} \overline{a^{-1}}$ as $\frac{\mathcal{C}}{a}$.

The regular solutions of (10) (see, for example, (50) and the comments following, or [KWCQ85, chaps. 2,3]) have an especially nice form. If $f$ and $g$ are holomorphic functions, we have

$$
\bar{\partial}_{k} f((1+k) z-(1-k) \bar{z})=0
$$

and

$$
\bar{\partial}_{k}\left(\frac{1+\frac{\lambda}{k}}{1+k} \overline{g(z)}-\frac{1-\frac{\lambda}{k}}{1-k} g(z)\right)=\widetilde{M} \overline{g^{\prime}(z)}
$$

Since $M$ and $\widetilde{M}$ are almost inverses of each other, in fact $M \widetilde{M}=4 \frac{\lambda}{k}$, we have for $k \neq 1$ the general form for regular solutions

$$
\begin{aligned}
w(z) & =f((1+k) z-(1-k) \bar{z})+\left(\frac{1+\frac{\lambda}{k}}{1+k}-\frac{1-\frac{\lambda}{k}}{1-k} \mathcal{C}\right) \overline{g(z)} \\
& =f((1+k) z-(1-k) \bar{z})+\frac{1+\frac{\lambda}{k}}{1+k} \overline{g(z)}-\frac{1-\frac{\lambda}{k}}{1-k} g(z) .
\end{aligned}
$$


When $k=1$ the form is slightly different (it can be obtained by taking the limit as $k \rightarrow 1):$

$$
w(z)=f(z)+\frac{1+\lambda}{2} \overline{g(z)}-\frac{1-\lambda}{2} \bar{z} g^{\prime}(z) .
$$

Summarizing some of the above, we will discuss in this paper strongly elliptic (Legendre-Hadamard) systems $L(\lambda, k)$ given by (10) with $0<k \leq 1$ and $k \leq \lambda$ of the following types:

(i) $L(\lambda, k), k \leq \lambda<1$, the nonsymmetric systems,

(ii) $L(k, k)=2 \partial \bar{\partial}_{k}, k<1$, the skewsymmetric systems,

(iii) $L(1, k)$, the triangular systems,

(iv) $L(\lambda, k), 1<\lambda$, the symmetrizable systems.

Special cases of the symmetrizable systems are $L(\lambda, 1)$ systems of the first kind (elastostatics systems) and $L(1,1)=4 \partial \bar{\partial}=\Delta$.

\section{Fundamental SOlution AND CONORMal DERIVATIVE}

In this section we construct the fundamental solution operator and use it to define an appropriate conormal derivative for solutions. With $\xi=\xi_{1}+i \xi_{2}$ and $|\xi|=1$ the symbol of $(10)$ is

$$
L(\xi)=\frac{k+\lambda}{4 k}\left((1+k)+(1-k) \bar{\xi}^{2}\right)+\frac{k-\lambda}{4 k}\left((1-k)+(1+k) \bar{\xi}^{2}\right) \mathcal{C} .
$$

To construct the fundamental solution we need the inverse operator (see Remark 4)

$$
L^{-1}(\xi)=\frac{(1+k / \lambda) \xi^{2}+(1-k / \lambda) \mathcal{C}}{(1+k) \xi^{2}+(1-k)}
$$

The fundamental solution operator may be obtained by computing the integral [Joh55, page 76]

$$
I(w)=\frac{1}{8 \pi^{2}} 4 \partial \bar{\partial} \int_{|\vec{\xi}|=1}(\Re(w \bar{\xi}))^{2} \log |\Re(w \bar{\xi})| L^{-1}(\xi) d \sigma(\vec{\xi}),
$$

where $\vec{\xi}=\left(\begin{array}{c}\xi_{1} \\ \xi_{2}\end{array}\right), \xi=\xi_{1}+i \xi_{2}, d \sigma$ denotes Lebesgue measure on the unit circle and $w$ is the complex notation for $\vec{y}-\vec{x}$. Taking the derivatives inside the integral, we have

$$
\begin{aligned}
I(w)=\frac{1}{4 \pi^{2}} \int_{|\vec{\xi}|=1}(\log |\Re(w \bar{\xi})|+3 / 2) L^{-1}(\xi) d \sigma(\vec{\xi}) & \\
= & \frac{1}{4 \pi^{2} i} \int_{|\xi|=1} \frac{d \xi}{\xi}(\log |\Re(w \bar{\xi})|+3 / 2) L^{-1}(\xi)
\end{aligned}
$$


in complex notation. Derivatives of $I(w)$ may now be computed by

$$
\begin{aligned}
\partial I(w)=\frac{1}{2 \pi} \partial_{w} & {\left[\frac{1}{2 \pi i} \int_{|\xi|=1} \frac{d \xi}{\xi} \log |\Re(w \bar{\xi})|\left(L^{-1}(\xi)-L^{-1}\left(\frac{i w}{|w|}\right)\right)\right.} \\
+ & \left.\left(\log |w|+\frac{1}{2 \pi i} \int_{|\xi|=1} \frac{d \xi}{\xi} \log \left|\Re \frac{w}{|w|} \bar{\xi}\right|\right) L^{-1}\left(\frac{i w}{|w|}\right)\right] \\
=\frac{1}{4 \pi} & {\left[\frac{1}{2 \pi i} \int_{|\xi|=1} \frac{d \xi}{\xi^{2} \Re(w \bar{\xi})}\left(L^{-1}(\xi)-L^{-1}\left(\frac{i w}{|w|}\right)\right)\right]+\frac{1}{4 \pi} \frac{1}{w} L^{-1}\left(\frac{i w}{|w|}\right) }
\end{aligned}
$$

and the residue theorem applied, rewriting $2 \Re(w \bar{\xi})=w / \xi+\bar{w} \xi$, to obtain

$$
\partial I(w)=\frac{1}{4 \pi w} L^{-1}\left(\frac{i w}{|w|}\right) .
$$

Similarly,

$$
\bar{\partial} I(w)=\frac{1+k / \lambda}{2 \pi(1+k) \bar{w}}-\frac{1}{4 \pi \bar{w}} L^{-1}\left(\frac{i w}{|w|}\right) .
$$

We remark that a useful form of $L^{-1}\left(\frac{i w}{|w|}\right)$ for calculation is

$$
L^{-1}\left(\frac{i w}{|w|}\right)=\frac{(1+k / \lambda) w-\bar{w}(1-k / \lambda) \mathcal{C}}{(1+k) w-(1-k) \bar{w}} .
$$

The fundamental solution can now be obtained by integrating either (16) or (17). For $k \neq 1$

$$
\begin{gathered}
=\frac{1}{4 \pi}\left(\log \left(\frac{1+k}{2} \zeta-\frac{1-k}{2} \bar{\zeta}\right)\left[\frac{1+\frac{k}{\lambda}}{1+k}-\frac{1-\frac{k}{\lambda}}{1-k} \mathcal{C}\right]+\left[\frac{1+\frac{k}{\lambda}}{1+k}+\frac{1-\frac{k}{\lambda}}{1-k} \mathcal{C}\right] \log \bar{\zeta}\right) \\
=\frac{1}{4 \pi}\left(\frac{1+\frac{k}{\lambda}}{1+k} \log |\zeta|^{2}+\log \left(\frac{1+k}{2}-\frac{1-k}{2} \frac{\bar{\zeta}}{\zeta}\right)\left[\frac{1+\frac{k}{\lambda}}{1+k}-\frac{1-\frac{k}{\lambda}}{1-k} \mathcal{C}\right]\right)
\end{gathered}
$$

using the principal branch of the logarithm. For $k=1$

$$
\Gamma(\zeta)=\frac{1}{8 \pi}\left(\left(1+\frac{1}{\lambda}\right) \log |\zeta|^{2}+\left(1-\frac{1}{\lambda}\right) \frac{\bar{\zeta}}{\zeta} \mathcal{C}\right)
$$

In either case $L \Gamma(\zeta)=\delta_{0}(\zeta)$, the Dirac $\delta$-operator at the origin.

Remark 5. If $f$ and $g$ are holomorphic functions, a solution operator of the same form, up to multiplication by constants, as $\Gamma$ may be defined by

$$
f((1+k) \zeta-(1-k) \bar{\zeta})[M-k \widetilde{M}]+[\widetilde{M}-k M] \overline{g(\zeta)}
$$

Compare with the solution function given by (12).

Our next task is to define an appropriate conormal derivative. Let $\Omega$ be any bounded Lipschitz domain in the plane, i.e. for any point $P \in \partial \Omega$ there is a neighborhood $U$ of $P$, a Lipschitz function $\phi: \mathbb{R} \rightarrow \mathbb{R}$ and a rectangular coordinate system $(s, t)$ so that $U \cap \Omega=U \cap\{(s, t) \mid t>\phi(s)\}$. Here $\phi$ being Lipschitz means $\left|\phi\left(s_{1}\right)-\phi\left(s_{2}\right)\right| \leq b\left|s_{1}-s_{2}\right|$ for all $s_{1}, s_{2}$ and some $b<\infty$. The outer unit normal vector $\vec{N}_{P}=\vec{N}=\left(\begin{array}{l}N_{1} \\ N_{2}\end{array}\right)$ is defined a.e. with respect to surface measure $d \sigma(P)$ on 
$\partial \Omega$. Let $N=N_{1}+i N_{2}$. For each operator $L=\partial M \bar{\partial}_{k}$ we define the conormal derivative at the boundary by

$$
\frac{\partial}{\partial \nu}=\frac{\bar{N}}{2} M(k, \lambda) \bar{\partial}_{k}-i \frac{\partial}{\partial \tau},
$$

where the tangential derivative $\partial / \partial \tau$ is defined by

$$
\frac{\partial}{\partial \tau}=-i \frac{1+k}{1+\frac{k}{\lambda}}(\overline{N \partial}-N \partial)
$$

The conormal derivatives (21) are those of [DL92, p. 659]. The tangential derivatives appear in (21) for the same reason as in [DKV88, p. 812]. On a flat boundary the trace of $\frac{\partial \Gamma}{\partial \nu}$ will have no tangential operators, i.e. Hilbert transforms, and will therefore be precisely $\frac{1}{2} \delta_{0}$. Consequently, on a boundary of class $C^{1}$ or better, $\frac{\partial \Gamma}{\partial \nu}$ will have the trace $\frac{1}{2} \delta_{0}$ plus compact operators, just as in [FJR78]. To determine the constant $\frac{1+k}{1+\frac{k}{\lambda}}$ in $(22)$, consider a piece of flat boundary containing both the origin and $\zeta$. The normal at $\zeta$ is then in the direction of $i \zeta$. Now apply the operator $\frac{\bar{N}}{2} M \bar{\partial}_{k}$ to $\Gamma(\zeta-0)$ at the boundary point $\zeta$ and subtract off $(\overline{N \partial}-N \partial)$ derivatives in order to get $\frac{\partial}{\partial \nu} \Gamma(\zeta)=0($ see $(28))$.

\section{Potential theory}

We develop Green's formulas and then define double and single layer potentials. First we need an integration by parts formula. Recall the operators $M$ and $\widetilde{M}$ and the representation $L=\partial \bar{\partial}(M+k \widetilde{M})+\partial^{2}(M-k \widetilde{M})$ from (11). We note that $M(i u)=i \widetilde{M} u$, and when considering products of functions operated upon by $M$ we also note that $\Re(v M u)=\Re(u M v)$ and $\Im(v M u)=\Im(u \widetilde{M} v)$.

Theorem 4.1 (Integration by parts). Given sufficiently smooth functions $u, v$ and boundary $\partial \Omega$ for a bounded domain $\Omega$,

$$
\begin{aligned}
& \Re \int_{\Omega} L u(M-k \widetilde{M}) v-(M-k \widetilde{M}) u L v d x d y \\
& \quad=\Re \int_{\partial \Omega} \frac{\partial u}{\partial \nu}(M-k \widetilde{M}) v-(M-k \widetilde{M}) u \frac{\partial v}{\partial \nu} d \sigma .
\end{aligned}
$$

Proof. The left side of (23) equals

$$
\begin{aligned}
& \Re \int_{\partial \Omega} \frac{\bar{N}}{2} M \overline{\partial_{k}} u(M-k \widetilde{M}) v-(M-k \widetilde{M}) u \frac{\bar{N}}{2} M \overline{\partial_{k}} v d \sigma \\
& \quad-\Re \int_{\Omega} \bar{\partial}(M+k \widetilde{M}) u \partial(M-k \widetilde{M}) v-\partial(M-k \widetilde{M}) u \bar{\partial}(M+k \widetilde{M}) v d x d y
\end{aligned}
$$


Writing out the solid integral, we get

$$
\begin{aligned}
\Re \int_{\Omega} \bar{\partial}( & \left.(1+k)\left(1+\frac{\lambda}{k}\right)+(1-k)\left(1-\frac{\lambda}{k}\right) \mathcal{C}\right) u \\
& \times \partial\left((1-k)\left(1+\frac{\lambda}{k}\right)+(1+k)\left(1-\frac{\lambda}{k}\right) \mathcal{C}\right) v \\
-\partial & \left((1-k)\left(1+\frac{\lambda}{k}\right)+(1+k)\left(1-\frac{\lambda}{k}\right) \mathcal{C}\right) u \\
& \times \bar{\partial}\left((1+k)\left(1+\frac{\lambda}{k}\right)+(1-k)\left(1-\frac{\lambda}{k}\right) \mathcal{C}\right) v d x d y \\
= & \Re\left(1-k^{2}\right) \int_{\Omega}\left(1+\frac{\lambda}{k}\right)^{2}(\bar{\partial} u \partial v-\partial u \bar{\partial} v)+\left(1-\frac{\lambda}{k}\right)^{2}(\bar{\partial} \bar{u} \partial \bar{v}-\partial \bar{u} \bar{\partial} \bar{v}) d x d y
\end{aligned}
$$

Continuing:

$$
\begin{aligned}
& =\Re 4 \frac{\lambda}{k}\left(1-k^{2}\right) \int_{\Omega} \bar{\partial} u \partial v-\partial u \bar{\partial} v d x d y \\
& =\Re \frac{\lambda}{k}\left(1-k^{2}\right) \int_{\partial \Omega}(\overline{N \partial}-N \partial) u v-u(\overline{N \partial}-N \partial) v d \sigma \\
& =\Re \frac{1+k}{\frac{k}{\lambda}+1} \int_{\partial \Omega}(\overline{N \partial}-N \partial) u(1-k)\left(1+\frac{\lambda}{k}\right) v-(1-k)\left(1+\frac{\lambda}{k}\right) u(\overline{N \partial}-N \partial) v d \sigma \\
& =\Re \frac{1+k}{\frac{k}{\lambda}+1} \int_{\partial \Omega}(\overline{N \partial}-N \partial) u(M-k \widetilde{M}) v-(M-k \widetilde{M}) u(\overline{N \partial}-N \partial) v d \sigma .
\end{aligned}
$$

Subtracting from the boundary integral in (24) yields the formula.

Thinking of the fundamental solution operators (19) and (20) above as matrices, one may, by Remark 4, form functions from the columns. For (19)

$$
\Gamma_{1}(\zeta)=\frac{1}{4 \pi}\left\{\frac{1+\frac{k}{\lambda}}{1+k} \log |\zeta|^{2}+\left[\frac{1+\frac{k}{\lambda}}{1+k}-\frac{1-\frac{k}{\lambda}}{1-k}\right] \log \left(\frac{1+k}{2}-\frac{1-k}{2} \frac{\bar{\zeta}}{\zeta}\right)\right\}
$$

and

$$
\Gamma_{2}(\zeta)=\frac{i}{4 \pi}\left\{\frac{1+\frac{k}{\lambda}}{1+k} \log |\zeta|^{2}+\left[\frac{1+\frac{k}{\lambda}}{1+k}+\frac{1-\frac{k}{\lambda}}{1-k}\right] \log \left(\frac{1+k}{2}-\frac{1-k}{2} \frac{\bar{\zeta}}{\zeta}\right)\right\}
$$

For $(20)$

$$
\Gamma_{1}(\zeta)=\frac{1}{8 \pi}\left\{\left(1+\frac{1}{\lambda}\right) \log |\zeta|^{2}+\left(1-\frac{1}{\lambda}\right) \frac{\bar{\zeta}}{\zeta}\right\}
$$

and

$$
\Gamma_{2}(\zeta)=\frac{i}{8 \pi}\left\{\left(1+\frac{1}{\lambda}\right) \log |\zeta|^{2}-\left(1-\frac{1}{\lambda}\right) \frac{\bar{\zeta}}{\zeta}\right\} .
$$

In either case we have

$$
L \Gamma=\delta_{0}, \quad L \Gamma_{1}=\delta_{0} \quad \text { and } \quad L \Gamma_{2}=i \delta_{0}
$$

where the first Dirac $\delta$ is an operator (matrix) while the second and third are thought of as "functions". 
Using the conormal derivative (21) we also have

$$
\frac{\partial}{\partial \nu} \Gamma(\zeta)=\frac{1}{4 \pi} \frac{1+k}{\frac{k}{\lambda}+1}\left(\frac{\bar{N}}{\bar{\zeta}}+\frac{N}{\zeta}\right) L^{-1}\left(\frac{i \zeta}{|\zeta|}\right)
$$

where $L^{-1}\left(\frac{i \zeta}{|\zeta|}\right)$ is as in (18).

The first and second columns are

$$
\begin{aligned}
& \left(\frac{\partial}{\partial \nu} \Gamma\right)_{1}(\zeta)=\frac{1}{4 \pi} \frac{1+k}{\frac{k}{\lambda}+1}\left(\frac{\bar{N}}{\bar{\zeta}}+\frac{N}{\zeta}\right) \frac{\left(\frac{k}{\lambda}+1\right) \zeta+\left(\frac{k}{\lambda}-1\right) \bar{\zeta}}{(1+k) \zeta-(1-k) \bar{\zeta}} \\
& \left(\frac{\partial}{\partial \nu} \Gamma\right)_{2}(\zeta)=\frac{i}{4 \pi} \frac{1+k}{\frac{k}{\lambda}+1}\left(\frac{\bar{N}}{\bar{\zeta}}+\frac{N}{\zeta}\right) \frac{\left(\frac{k}{\lambda}+1\right) \zeta-\left(\frac{k}{\lambda}-1\right) \bar{\zeta}}{(1+k) \zeta-(1-k) \bar{\zeta}}
\end{aligned}
$$

Another convenient form for the conormal of $\Gamma$ is

$$
\begin{aligned}
\frac{\partial}{\partial \nu} \Gamma(\zeta)= & \frac{1}{4 \pi(1-k)\left(\frac{\lambda}{k}+1\right)} \\
& \times\left\{\frac{(1+k) N+(1-k) \bar{N}}{(1+k) \zeta-(1-k) \bar{\zeta}}(M-k \widetilde{M})+(\widetilde{M}-k M) \frac{\bar{N}}{\bar{\zeta}}\right\} .
\end{aligned}
$$

Compare with (19) and Remark 5. Next we note that $M-k \widetilde{M}$ is invertible as long as $\lambda \neq 1$, i.e., for all but triangular systems. Furthermore,

$$
(M-k \widetilde{M})^{-1}=\frac{1}{\left(\frac{\lambda}{k}-k\right)(1-\lambda)}(\widetilde{M}-k M) .
$$

Theorem 4.2 (Green's formula). For $v$ and $\Omega$ as in Theorem 4.1, suppose $L v=$ w. Then

(i)

$$
\begin{aligned}
\Re(M-k \widetilde{M}) v(\xi) & \\
=\Re \int_{\partial \Omega} \frac{\partial \Gamma_{1}}{\partial \nu}(\zeta-\xi)(M-k \widetilde{M}) v(\zeta) & -\Gamma_{1}(\zeta-\xi)(M-k \widetilde{M}) \frac{\partial v}{\partial \nu}(\zeta) d \sigma(\zeta) \\
& +\Re \int_{\Omega} \Gamma_{1}(\zeta-\xi)(M-k \widetilde{M}) w(\zeta) d x d y
\end{aligned}
$$

(ii)

$$
\begin{array}{r}
\Im(M-k \widetilde{M}) v(\xi) \\
=-\Re \int_{\partial \Omega} \frac{\partial \Gamma_{2}}{\partial \nu}(\zeta-\xi)(M-k \widetilde{M}) v(\zeta)-\Gamma_{2}(\zeta-\xi)(M-k \widetilde{M}) \quad \frac{\partial v}{\partial \nu}(\zeta) d \sigma(\zeta) \\
-\Re \int_{\Omega} \Gamma_{2}(\zeta-\xi)(M-k \widetilde{M}) w(\zeta) d x d y
\end{array}
$$

(iii)

$$
\begin{aligned}
(M-k \widetilde{M}) v(\xi) & \\
=\int_{\partial \Omega} \overline{\frac{\partial \Gamma}{\partial \nu}(\zeta-\xi)}^{T}(M-k \widetilde{M}) v(\zeta)- & \overline{\Gamma(\zeta-\xi)}^{T}(M-k \widetilde{M}) \frac{\partial v}{\partial \nu}(\zeta) d \sigma(\zeta) \\
& +\int_{\Omega} \overline{\Gamma(\zeta-\xi)}^{T}(M-k \widetilde{M}) w(\zeta) d x d y
\end{aligned}
$$


(iv)

$$
v(\xi)=\int_{\partial \Omega} \frac{\partial \Gamma}{\partial \nu}(\zeta-\xi) v(\zeta)-\Gamma(\zeta-\xi) \frac{\partial v}{\partial \nu}(\zeta) d \sigma(\zeta)+\int_{\Omega} \Gamma(\zeta-\xi) w(\zeta) d x d y .
$$

Proof. Parts (i) and (ii) follow by the integration by parts formula (23) with $u=\Gamma_{1}$ or $-\Gamma_{2}$ together with (27). Part (iii) combines (i) and (ii) in an operator or matrix version, where $T$ stands for transposition of matrices. See Remark 4.

Part (iv) follows from (29) and the formulas $(\widetilde{M}-k M){\frac{\overline{\partial \Gamma}^{T}}{\partial \nu}}^{T}=\frac{\partial \Gamma}{\partial \nu}(\widetilde{M}-k M)$ and $(\widetilde{M}-k M) \bar{\Gamma}^{T}=\Gamma(\widetilde{M}-k M)$. These follow from the Hermitian formulas (see Remark 4)

$$
\overline{\frac{\partial \Gamma}{\partial \nu}(\widetilde{M}-k M)}^{T}=\frac{\partial \Gamma}{\partial \nu}(\widetilde{M}-k M) \text { and } \overline{\Gamma(\widetilde{M}-k M)}^{T}=\Gamma(\widetilde{M}-k M) .
$$

To see (30) we note that if $a$ and $b$ are real and $G$ is any complex valued function, then $(a+b \mathcal{C}) G(a+b \mathcal{C})$ is Hermitian. Because $M-k \widetilde{M}$ and $\widetilde{M}-k M$ are almost inverses of one another (29), it follows that anything of the form

$$
[F(M-k \widetilde{M})+(\widetilde{M}-k M) G](\widetilde{M}-k M)
$$

is Hermitian. Therefore (30) follows by Remark 5 and (28).

Part (iv) of Theorem 4.4 suggests that we may define for any $1<p<\infty$ and complex valued $f \in L^{p}(\partial \Omega)$ the double layer potential

$$
\mathcal{K} f(\xi)=\int_{\partial \Omega} \frac{\partial \Gamma}{\partial \nu}(\zeta-\xi) f(\zeta) d \sigma(\zeta), \quad \xi \in \mathbb{C} \backslash \partial \Omega,
$$

and the single layer potential

$$
\mathcal{S} f(\xi)=\int_{\partial \Omega} \Gamma(\zeta-\xi) f(\zeta) d \sigma(\zeta), \quad \xi \in \mathbb{C} .
$$

(27) shows that both $\mathcal{K} f$ and $\mathcal{S} f$ will be solutions to $L u=0$.

The definitions of $\mathcal{K}$ and $\mathcal{S}$ and their traces on the boundary make sense even on Lipschitz domains. This follows by well known arguments based on [Cal77], [CMM82] and [FJR78]. Recall the nontangential maximal function $\mathcal{N}(u)$ of a function $u$ defined in a Lipschitz domain $\Omega$. If $P \in \partial \Omega$,

$$
\mathcal{N}(u)(P)=\sup _{\zeta}|u(\zeta)|,
$$

where the supremum is taken over the nontangential approach region

$$
\{\zeta \in \Omega:|\zeta-P| \leq(1+\alpha) \operatorname{dist}(\zeta, \partial \Omega)\},
$$

where $\alpha>0$ is independent of $P$ but dependent on the Lipschitz nature of $\Omega$. The nontangential limit will be written $u(\zeta) \stackrel{\text { n.t. }}{\rightarrow} u(P)$ and is $\lim _{\zeta \rightarrow P} u(\zeta)$, where for each $P$ the $\zeta$ are taken in the region (33). A similar region may be constructed for $\bar{\Omega}^{c}$ and both $\mathcal{N}(u)$ and $\stackrel{\text { n.t. }}{\rightarrow}$ defined. We have by [CMM82] and [FJR78]

Theorem 4.3. Let $\Omega \subset \mathbb{C}$ be a Lipschitz domain. Let $1<p<\infty$ and $f \in L^{p}(\partial \Omega)$. Then

(i) $\|\mathcal{N}(\mathcal{K} f)\|_{L^{p}(\partial \Omega)} \leq C_{p}\|f\|_{L^{p}(\partial \Omega)}$,

(ii) $\mathcal{K} f(\zeta) \stackrel{\text { n.t. }}{\rightarrow}\left(\frac{1}{2} I+K\right) f(P)$ a.e. $(d \sigma), \zeta \in \Omega$, 
(iii) $\mathcal{K} f(\zeta) \stackrel{\text { n.t. }}{\rightarrow}\left(-\frac{1}{2} I+K\right) f(P)$ a.e. $(d \sigma), \zeta \in \bar{\Omega}^{c}$,

where $I$ is the identity operator, and

(iv) $K f(P)=p \cdot v \cdot \int_{\partial \Omega} \frac{\partial \Gamma}{\partial \nu}(\zeta-P) f(\zeta) d \sigma(\zeta)$ is bounded from $L^{p}(\partial \Omega)$ to $L^{p}(\partial \Omega)$

and $\frac{\partial}{\partial \nu}$ is defined by (21) with the outer unit normals $N(\zeta)$ to $\partial \Omega$.

We define an adjoint to $K$ by

$$
K^{*} f(P)=p \cdot v \cdot \int_{\partial \Omega} \frac{\partial \Gamma}{\partial \nu_{P}}(P-\zeta) f(\zeta) d \sigma(\zeta)
$$

where $\frac{\partial}{\partial \nu_{P}}$ is defined by (21) with the outer normals $N(P)$ to $\partial \Omega$.

We obtain by the observations (30) (see Remark 4) and (29)

$$
\Re \int_{\partial \Omega}(M-k \widetilde{M}) g K f d \sigma=\Re \int_{\partial \Omega}(M-k \widetilde{M}) f K^{*} g d \sigma
$$

and

Theorem 4.4. With the same hypotheses as in Theorem 4.3

(i) $\|\mathcal{N}(|\partial \mathcal{S} f|+|\bar{\partial} \mathcal{S} f|)\|_{L^{p}(\partial \Omega)} \leq C_{p}\|f\|_{L^{p}(\partial \Omega)}$,

(ii) $\frac{\partial}{\partial \nu_{P}} \mathcal{S} f(\zeta) \stackrel{\text { n.t. }}{\rightarrow}\left(-\frac{1}{2} I+K^{*}\right) f(P)$ a.e. $(d \sigma), \zeta \in \Omega$,

(iii) $\frac{\partial}{\partial \nu_{P}} \mathcal{S} f(\zeta) \stackrel{\text { n.t. }}{\rightarrow}\left(\frac{1}{2} I+K^{*}\right) f(P)$ a.e. $(d \sigma), \zeta \in \bar{\Omega}^{c}$,

(iv) $K^{*}$ is bounded from $L^{p}(\partial \Omega)$ to $L^{p}(\partial \Omega)$.

Remark 6. One of our goals is to show on Lipschitz boundaries that $\frac{1}{2} I+K^{*}$ is injective from $L^{p}$ to $L^{p}$, where $p>1$ is chosen small enough, depending on the canonical ellipticity $k$. By duality this implies that $\frac{1}{2} I+K$ has dense range in $L^{p^{\prime}}$. As is apparent, our operators are not linear over complex $L^{p}$ spaces. For example, (34) is not true when $\Im$ replaces $\Re$, so that $(M-k \widetilde{M}) K^{*}$ is not the adjoint of $(M-k \widetilde{M}) K$ over complex $L^{p}$. The algebraic convenience of our notation seems to cause an analytic difficulty. However, if our operators are rewritten as acting on two copies of real $L^{p}$, one sees that they then are linear and, for example, $(M-k \widetilde{M}) K^{*}$ is the adjoint to $(M-k \widetilde{M}) K$. In this way standard functional analytic arguments can be seen to be valid.

\section{Spectral analysis}

As in [DL92], determining that the trace of the double layer potential on the boundary of a $C^{1}$ polygon is a Fredholm operator can be reduced to analyzing the spectrum of the trace in unbounded plane sectors. However, in general it is not enough to match the sectors' angles with the angles of the polygon. In addition the orientation of the angles must also match. For canonical ellipticity $k<1$ the spectrum of the trace of the double layer potential is not rotation invariant. Thus we will give a brief derivation of the symbol associated with the trace that takes this view into account. 
Let $\alpha, \beta \in \mathbb{C}$ have norm 1 and be distinct. Define the sector

$$
\Omega_{\alpha, \beta}=\{\zeta \mid \arg \alpha<\arg \zeta<\arg \beta\} .
$$

Let $s, t$ be positive reals. Using the definition of the double layer potential $\mathcal{K},(31)$, and letting $\zeta \in \Omega_{\alpha, \beta}$ approach $t \beta$ as in (ii) of Theorem 4.3, we obtain the trace

$$
\left(\frac{1}{2} I+K\right) f(t \beta)=\frac{1}{2} f(t \beta)+\int_{0}^{\infty} \frac{\partial \Gamma}{\partial \nu}(s \alpha-t \beta) f(s \alpha) d s .
$$

Here $N=-i \alpha$ in $(21)$ and, putting $2 A=(1+k) \alpha-(1-k) \bar{\alpha}$ and $2 B=(1+k) \beta-$ $(1-k) \bar{\beta}$, the kernel of the integral operator becomes the Hardy kernel [LP80]

$$
K_{\alpha}(s, t)=\frac{1}{4 \pi i(1-k)\left(\frac{\lambda}{k}+1\right)}\left\{\frac{A}{A-\frac{t}{s} B}(M-k \widetilde{M})-(M-k \widetilde{M}) \frac{\bar{\alpha}}{\bar{\alpha}-\frac{t}{s} \bar{\beta}}\right\} \frac{1}{s} .
$$

Now define $\beta / \alpha=e^{i \theta}$; then $0<\theta<2 \pi$ represents the interior angle of the sector $\Omega_{\alpha, \beta}$. Define $\arg (B / A)=\psi, 0<\psi<2 \pi$. Then $\psi$ varies as the orientation $(\arg \alpha+\arg \beta) / 2$ varies. $B$ and $A$ are the horizontal projection of $\beta$ and $\alpha$ onto the ellipse $x^{2} / k^{2}+y^{2}=1$. When $k=1, \psi=\theta$, but when $k<1$ and $\theta$ is fixed $\psi$ varies as a function of orientation since it is the angle between the projections of $\alpha$ and $\beta$ onto the ellipse.

Using (1.2) and (1.6) of [LP80], we obtain via the Mellin transform the symbol for $K_{\alpha}$ (see 5.27 of [LP83]):

$$
\begin{array}{r}
\widetilde{k}_{\alpha}(z) \\
=\frac{1}{4 \pi i(1-k)\left(\frac{\lambda}{k}+1\right)}\left\{\left|\frac{A}{B}\right|^{z} e^{-i \psi} \frac{e^{i \pi z}}{\sin \pi z}(M-k \widetilde{M})-(M-k \widetilde{M}) e^{i \theta z} \frac{e^{-i \pi z}}{\sin \pi z}\right\} \\
=\frac{1}{4 i \sin \pi z}\left\{\left|\frac{B}{A}\right|^{-z} e^{z(\pi-\psi)}[1+\Lambda \mathcal{C}]-[1+\Lambda \mathcal{C}] e^{-i z(\pi-\theta)}\right\},
\end{array}
$$

where $0<\Re z<1$ and $\Lambda=\frac{(1+k)\left(1-\frac{\lambda}{k}\right)}{(1-k)\left(1+\frac{\lambda}{k}\right)}$.

Remark 7 . For the time being, however, $z$ should be thought of as an indeterminate rather than as a complex number. The conjugation operators in $M$ and the other complex algebraic operations we use do not affect $z$. Our use of the algebra of complex numbers is to facilitate algebraic manipulations of our real systems and their solutions. With $z$ we have introduced a separate complex structure. For example, $\mathcal{C} z=z$ and $\mathcal{C} e^{i z}=e^{-i z}$.

Similarly we let $\zeta \in \Omega_{\alpha, \beta}$ approach $t \alpha$, obtaining

$$
\left(\frac{1}{2} I+K\right) f(t \alpha)=\frac{1}{2} f(t \alpha)+\int_{0}^{\infty} K_{\beta}(s, t) f(s \beta) d s,
$$

and find that the symbol of the Hardy kernel $K_{\beta}$ satisfies $\widetilde{k}_{\beta}(z)=\widetilde{k}_{\alpha}(-z)$. Writing $f_{\alpha}(s)=f(s \alpha)$ and $f_{\beta}(s)=f(s \beta)$, the problem of inverting the trace of the double layer potential on $\partial \Omega_{\alpha, \beta}$ is the same as solving the system

$$
\left(\frac{1}{2} I+K\right) f=\left(\begin{array}{cc}
\frac{1}{2} I & K_{\beta} \\
K_{\alpha} & \frac{1}{2} I
\end{array}\right)\left(\begin{array}{c}
f_{\alpha} \\
f_{\beta}
\end{array}\right)=\left(\begin{array}{c}
g_{\alpha} \\
g_{\beta}
\end{array}\right)
$$


where the complex valued functions $f_{\alpha}, f_{\beta}, g_{\alpha}, g_{\beta} \in L^{p}\left(\mathbb{R}_{+}\right)$. This can be done as in [DL92] by studying the matrix of symbols

$$
2\left(\begin{array}{cc}
\frac{1}{2} & \widetilde{k}_{\alpha}(-z) \\
\widetilde{k}_{\alpha}(z) & \frac{1}{2}
\end{array}\right)
$$

and in particular its "determinant" symbols

$$
4 \widetilde{k}_{\alpha}(z) \widetilde{k}_{\alpha}(-z)-1 \text { and } 4 \widetilde{k}_{\alpha}(-z) \widetilde{k}_{\alpha}(z)-1
$$

The first symbol of (39) factors as

$$
\begin{aligned}
& 4 \widetilde{k}_{\alpha}(z) \widetilde{k}_{\alpha}(-z)-1 \\
& =\frac{1}{\sin ^{2} \pi z}\left[\sin \frac{z}{2}\left(4 \pi-\theta-\psi+i \log \left|\frac{B}{A}\right|\right)+\Lambda e^{i z(2 \pi-\psi)} \sin \frac{z}{2}\left(\theta-\psi+i \log \left|\frac{B}{A}\right|\right) \mathcal{C}\right] \\
& \quad \times\left[\sin \frac{z}{2}\left(-\theta-\psi+i \log \left|\frac{B}{A}\right|\right)+\Lambda e^{i z \theta} \sin \frac{z}{2}\left(\theta-\psi+i \log \left|\frac{B}{A}\right|\right) \mathcal{C}\right]
\end{aligned}
$$

and similarly for the second. The case $k=1$ is obtained in the limit, viz.

$$
\begin{aligned}
4 \widetilde{k}_{\alpha}(z) \widetilde{k}_{\alpha}(-z)-1 & \\
=\frac{1}{\sin ^{2} \pi z}[\sin z(2 \pi-\theta)+ & \left.i \frac{1-\lambda}{1+\lambda} z \frac{\beta^{2}-\alpha^{2}}{2} e^{i z(2 \pi-\theta)} \mathcal{C}\right] \\
& \times\left[-\sin z \theta+i \frac{1-\lambda}{1+\lambda} z \frac{\beta^{2}-\alpha^{2}}{2} e^{i z \theta} \mathcal{C}\right] .
\end{aligned}
$$

Finally the determinant of (38) as a $4 \times 4$ matrix follows from the determinant (see Remark 4) of the symbols (39) in the $k<1$ case (recall that $z$ is not treated as a complex number in this calculation) as

$$
\begin{aligned}
\frac{1}{\sin ^{4} \pi z} & {\left[\left|\sin \frac{z}{2}\left(4 \pi-\theta-\psi+i \log \left|\frac{B}{A}\right|\right)\right|^{2}-\Lambda^{2}\left|\sin \frac{z}{2}\left(\theta-\psi+i \log \left|\frac{B}{A}\right|\right)\right|^{2}\right] } \\
\times & {\left[\left|\sin \frac{z}{2}\left(\theta+\psi-i \log \left|\frac{B}{A}\right|\right)\right|^{2}-\Lambda^{2}\left|\sin \frac{z}{2}\left(\theta-\psi+i \log \left|\frac{B}{A}\right|\right)\right|^{2}\right] } \\
=\frac{1}{\sin ^{4} \pi z} & {\left[\cos i z \log \left|\frac{B}{A}\right|-\cos z(4 \pi-\theta-\psi)-\Lambda^{2}\left(\cos i z \log \left|\frac{B}{A}\right|-\cos z(\theta-\psi)\right)\right] } \\
\times & {\left[\cos i z \log \left|\frac{B}{A}\right|-\cos z(\theta+\psi)-\Lambda^{2}\left(\cos i z \log \left|\frac{B}{A}\right|-\cos z(\theta-\psi)\right)\right] }
\end{aligned}
$$

and in the $k=1$ case as

$$
\begin{aligned}
\frac{1}{\sin ^{4} \pi z} & {\left[\sin ^{2} z(2 \pi-\theta)-\left(\frac{1-\lambda}{1+\lambda}\right)^{2} z^{2} \sin ^{2} \theta\right] } \\
\times & {\left[\sin ^{2} z \theta-\left(\frac{1-\lambda}{1+\lambda}\right)^{2} z^{2} \sin ^{2} \theta\right] }
\end{aligned}
$$

The formulas (40) and (41) are, in fact, Theorem 4.1 of [DL92, pp. 662-663]. When $1<p<\infty$ the operator $\frac{1}{2} I+K(37)$ will be invertible on $L^{p}\left(\partial \Omega_{\alpha, \beta}\right)$ as long as there is no $z=\frac{1}{p}+i \xi$ for $\xi \in \mathbb{R}$ for which the determinant (40) vanishes. By Theorem 4.3 of [DL92] the symmetrizable $(\lambda>1)$ systems have double layer potentials that 
fail to be invertible for some sector only when $1<p<2$. This is consistent with the existence of Rellich formulas ([PV95a], [Ver96], and Remark 8 below) for such systems, thus insuring $L^{2}$ results and the $L^{p}$ results for $p>2$. Further, the zeros of (40) are always of the form $z=\frac{1}{p}$, i.e. real, and at most two in number (when an angle $\theta$ and orientation are fixed).

In the case of nonsymmetric systems $k \leq \lambda<1$ (40) begins to have nonreal zeros in the strip $0<\Re z<1$, and they begin to have the form $z=\frac{1}{p}+i \xi, p \geq 2$. This is most readily seen in the skewsymmetric case $\lambda=k<1$. In this case $\Lambda=0$ and, for example, a zero may satisfy

$$
\Re z=\frac{1}{p}=\frac{\frac{n}{2} \frac{\theta+\psi}{4 \pi}}{\left(\frac{\theta+\psi}{4 \pi}\right)^{2}+\left(\frac{1}{4 \pi} \log \left|\frac{B}{A}\right|\right)^{2}}
$$

$n=1,2, \ldots ; 0<\theta, \psi<2 \pi$. Then an example of $p=2$ noninvertibility (see $\S 8$ ) is obtained by taking $n=1, \alpha=1$, and $\beta=i$ ( $\operatorname{so} \theta=\psi=\pi / 2$ ) and $k=e^{-\pi \sqrt{3}}$ (since now $\log \left|\frac{B}{A}\right|=\log (1 / k)$ ).

In fact by choosing $k$ small and thus allowing $\log \left|\frac{B}{A}\right|$ to be as large as $\log (1 / k)$ it is clear from (42) that noninvertibility may occur for angles $\theta$ in the vicinity of $\pi / 2$ and orientation in the vicinity of $\pi / 4$ for as large a (finite) number of values of $p$ as we wish. Further, invertibility may be made to fail for any $p<\infty$ by choosing $k$ small enough.

As with the Laplacian [FJL77], failure of the double layer potential to be invertible does not necessarily imply inability to solve the Dirichlet problem. As is apparent, however, the zeros of the second factor of (40) correspond exactly to the counterexamples in $\S 8$. Thus other observations from $\S 8$ apply here. The first factor of (40) allows additional noninvertibility results even when the Dirichlet problem is solvable. See $\S 6$ below.

We close with an observation on systems of the first kind $(k=1)$ and a lemma which lays the foundation for the positive results in $C^{1}$ polygons of $\S \S 6$ and 7 .

Theorem 5.1. The noninvertibility of the trace of the double layer potential from $L^{p}(\partial \Omega)$ to $L^{p}(\partial \Omega), 1<p<\infty$, when $\Omega$ is a sector (35) is invariant under rotations of the sector when $k=1$.

When $k<1$ invertibility fails on nontrivial closed intervals of p's for a fixed interior angle $\theta$ as the sector is rotated.

The proof follows immediately from formulas (41) and (40) and the symbol calculus of [LP80].

In the lemma, strong ellipticity, $\lambda>0$, is used for the first time. See Remarks 1 and 2 .

Lemma 5.1. Given a strongly elliptic system with $k$ and $\lambda$, there is a $p_{0}^{\prime}<\infty$ so that on every sector $\Omega_{\alpha, \beta}$ (35) the trace of the double layer potential (37) is invertible on $L^{p}\left(\partial \Omega_{\alpha, \beta}\right)$ for all $p_{0}^{\prime} \leq p<\infty$.

Proof. When $\lambda \geq 1$ [DL92] shows that we may take $p_{0}^{\prime}=2$. We need only consider the nonsymmetric case $k \leq \lambda<1$. In this case $0 \leq \Lambda^{2}<1$, where $\Lambda$ is as in (36). 
Let $z=x+i y$ and let $\rho=\log \left|\frac{B}{A}\right|$. We will call $\sin ^{4} \pi z$ the denominator of $(40)$; then the negative of the real part of the second factor in the numerator of (40) may be written as

$$
\begin{aligned}
& \left(1-\Lambda^{2}\right)[\cos x(\theta+\psi) \cosh y(\theta+\psi)-\cos y \rho \cosh x \rho] \\
& \quad+\Lambda^{2}[\cos x(\theta+\psi) \cosh y(\theta+\psi)-\cos x(\theta-\psi) \cosh y(\theta-\psi)] .
\end{aligned}
$$

We will first show that (43) never vanishes for any $0<\theta<2 \pi$ whenever

$$
z \in\left\{x+i y \mid 0 \leq x \leq 2^{-4} \text { and }|y|>\frac{1}{k}\right\} .
$$

It is always true that $|\rho| \leq \log \left(1+\frac{|B-A|}{k}\right) \leq \frac{\theta}{k}$ and $0 \leq \theta+\psi \leq 4 \pi$. Thus, setting $t=\theta+\psi$, the first bracket of (43) is bounded below by

$$
f(t)=\cos x t \cosh y t-\cosh \frac{x}{k} t \text {. }
$$

By examining $f^{\prime \prime}(t)$ it is not hard to see, with $x$ and $y$ as in $(44)$, that $f(t)$ is an increasing function of $t, 0 \leq t \leq 4 \pi$. Thus the first bracket of (43) is positive for $0<\theta<2 \pi$, and so is the second since $f(t)+\cosh \frac{x}{k} t$ is also increasing and $\theta+\psi>|\theta-\psi|$. Thus the second factor in the numerator of (40) has no zeros in the half-strips (44). A similar analysis holds for the first factor in the numerator of (40) when $0<\theta<2 \pi$, since $\rho \leq \frac{2 \pi-\theta}{k}$ and $4 \pi-\theta-\psi>|\theta-\psi|$. Now we need only show that (40) has no zeros in a bounded rectangular region around the origin.

First the value of $(40)$ at the origin is

$$
\begin{aligned}
(2 \pi)^{-4} & {\left[(4 \pi-\theta-\psi)^{2}+\rho^{2}-\Lambda^{2}\left((\theta-\psi)^{2}+\rho^{2}\right)\right] } \\
& \times\left[(\theta+\psi)^{2}+\rho^{2}-\Lambda^{2}\left((\theta-\psi)^{2}+\rho^{2}\right)\right],
\end{aligned}
$$

which is positive for all $0<\theta+\psi<4 \pi$. Next, analyze the factors in the numerator. The negative of the second factor for $z=i y$ is $\cosh y(\theta+\psi)-\cos y \rho-$ $\left.\Lambda^{2}(\cosh y(\theta-\psi)-\cos y \rho)\right)$, which is positive whenever $y \neq 0$ and $0<\theta+\psi \leq 4 \pi$. A similar statement is true for the first factor. By continuity and compactness we need only exclude the possibility that zeros can come arbitrarily close to the $y$-axis in a bounded region about the origin when $\theta \downarrow 0$ or $\theta \uparrow 2 \pi$. Hence, let $|z|<b<\infty$. By the series expansion for the sine, (40) for any $|z|<b$ is equal to the origin value (46) with $+O\left(|4 \pi-\theta-\psi|^{4}\right)$ as $\theta \uparrow 2 \pi$ and $+O\left(|\theta+\psi|^{4}\right)$ as $\theta \downarrow 0$ inserted in the first and second brackets respectively, with these asymptotic expressions controlled by a constant that depends only on $b$ and $k$. Thus for $\theta$ close enough to 0 the second factor in the numerator of (40) can have no zeros $z$ with $|z|<b$, and similarly for $\theta$ close enough to $2 \pi$ for the first factor, which suffices to prove the lemma.

\section{6. $L^{p}$ BOUNDARY VALUE PROBLEMS}

We turn our attention to the solution of boundary value problems with $L^{p}$ data in $C^{1}$ polygons. These domains are a subset of the set of bounded Lipschitz domains with connected boundary consisting of a finite union of $C^{1}$ curves. We combine the method of layer potentials based on Lemma 5.1 above with the coercivity result of Theorem 6.1 to solve the Neumann problem. A rotation invariant Cauchy-Riemann system is devised (Theorem 6.6) in order to deduce results for the regularity problem 
from the Neumann problem. The section ends with the study of the Green operator for $L$ and its application to the Dirichlet problem.

To begin we need another integration by parts formula. Again, one sees the need for strong ellipticity, $\lambda>0$.

Theorem 6.1. (Analytic integration by parts [KWCQ85, pp. 25-27]) With the hypotheses of Theorem 4.1 and $L$ as in (11) and writing $u=v+i w$ for $v, w$ real valued,

$$
\begin{aligned}
& \Re \int_{\partial \Omega} \frac{\partial u}{\partial \nu}(M-k \widetilde{M}) \bar{u} d \sigma-\Re \int_{\Omega} L u(M-k \widetilde{M}) \bar{u} d x d y \\
& \quad=2 \int_{\Omega}(1-\lambda) v_{x}^{2}+\lambda(1-\lambda) v_{y}^{2}+\frac{\lambda}{k^{2}}\left(\lambda-k^{2}\right) w_{x}^{2}+\left(\lambda-k^{2}\right) w_{y}^{2} d x d y
\end{aligned}
$$

and

$$
\begin{aligned}
& -\Re \int_{\partial \Omega} \frac{\partial u}{\partial \nu}(M-k \widetilde{M}) u d \sigma+\Re \int_{\Omega} L u(M-k \widetilde{M}) u d x d y \\
& \quad=2 \int_{\Omega}(\lambda-1) v_{x}^{2}+\lambda(\lambda-1) v_{y}^{2}+\frac{\lambda}{k^{2}}\left(\lambda-k^{2}\right) w_{x}^{2}+\left(\lambda-k^{2}\right) w_{y}^{2} \\
& \quad+(\lambda-1) \frac{\frac{\lambda}{k}-k}{\frac{\lambda}{k}+1} v_{x} w_{y}+(\lambda-1) \frac{\frac{\lambda}{k}-k}{\frac{k}{\lambda}+1} v_{y} w_{x} d x d y .
\end{aligned}
$$

Proof. The proof follows by the Gauss divergence theorem, writing everything in terms of real and imaginary parts and collecting terms.

Remark 8. (Rellich Identities) Fixing $N=N_{0}$ in (22), we obtain a fixed directional derivative $\frac{\partial}{\partial \tau_{0}}$ in the $i N_{0}$ direction. By defining $\Omega$ as the region above the graph of a Lipschitz function with respect to the direction $i N_{0}$ we mean precisely that $\Re\left(i \overline{N_{0}} N\right)$ is positive and uniformly bounded away from zero a.e. $(d \sigma)$, where here $N$ is the outer unit normal vector to $\Omega$. When we parametrize the subdomains of $\Omega$ that are obtained as translations of $\Omega$ in the $i N_{0}$ direction and then differentiate (48) with respect to the parameter, the coarea formula yields

$$
\begin{aligned}
\Re \int_{\partial \Omega} \frac{\partial}{\partial \nu}\left(\frac{\partial}{\partial \tau_{0}} u\right)(M-k \widetilde{M}) u+\frac{\partial}{\partial \nu} u(M-k \widetilde{M}) \frac{\partial}{\partial \tau_{0}} u d \sigma & \\
=2 \int_{\partial \Omega}( & (\lambda-1) v_{x}^{2}+\lambda(\lambda-1) v_{y}^{2}+\frac{\lambda}{k^{2}}\left(\lambda-k^{2}\right) w_{x}^{2}+\left(\lambda-k^{2}\right) w_{y}^{2} \\
& \left.+(\lambda-1) \frac{\frac{\lambda}{k}-k}{\frac{\lambda}{k}+1} v_{x} w_{y}+(\lambda-1) \frac{\frac{\lambda}{k}-k}{\frac{k}{\lambda}+1} v_{y} w_{x}\right) \frac{1+k}{1+\frac{k}{\lambda}} \Re\left(i \overline{N_{0}} N\right) d \sigma
\end{aligned}
$$

whenever $L u=0$. A Rellich identity for solutions is then obtained by observing that $\frac{\partial u}{\partial \tau_{0}}$ is also a solution, so that integration by parts (Theorem 4.1) may be used to convert the first term on the left into another second term.

For symmetrizable systems, i.e. $\lambda>1$, the identity yields $L^{2}(\partial \Omega)$ control of all first derivatives of a solution in terms of either the conormal derivative or the transverse derivative $\frac{\partial u}{\partial \tau_{0}}$, thus yielding $L^{2}(\partial \Omega)$ results for the Neumann and Dirichlet problems on Lipschitz domains (see [JK81], [PV95a] and others). When $k \leq \lambda<1$ the right side of the identity fails to control the $L^{2}(\partial \Omega)$ norm of the derivatives, and 
thus one considers deriving a similar identity using (47). But $\bar{u}$ is not a solution, so the integration by parts fails to yield a useful identity except in the case where $\lambda>k$ and the Lipschitz constant for $\partial \Omega$ is so small that the extra terms deriving from $L \bar{u}$ may be hidden. When $\lambda=k$ a Rellich identity, simply obtained as here by using the divergence theorem, appears to be impossible even in the flat boundary case.

Theorem 6.2 (Uniqueness for the Neumann problem). Let $\Omega \subset \mathbb{C}$ be a bounded Lipschitz domain. Let $1 \leq p \leq \infty$ and suppose $L u=0$ in $\Omega$ with

$$
\mathcal{N}(|\partial u|+|\bar{\partial} u|) \in L^{p}(\partial \Omega) .
$$

Then $\frac{\partial u}{\partial \nu}=0$ a.e. $(d \sigma)$ implies that $u$ is constant.

Proof. When $\lambda=1$, we have the triangular systems. Writing $u=v+i w$, we have that $w$ satisfies a single elliptic homogeneous equation with conormal derivative equal to zero. Thus by the theory of single elliptic equations in Lipschitz domains $w$ is constant, whence $v$ too satisfies the same type of equation and boundary conditions, and the proof is complete by the single equation theory.

When $k \leq \lambda<1$ we use the equation of (47) on domains approximating $\Omega$ from the inside, noting that (49) implies that $|u|$ is a uniformly bounded function in $\Omega$. Thus, by (47), $\frac{\partial u}{\partial \nu}=0$, and by the Lebesgue dominated convergence theorem the left side of (47) is in the limit zero. Thus $u$ is a constant.

When $\lambda>1$ we have symmetric systems, and the result follows from (48) and Young's inequality to take care of the cross terms.

By Theorem 4.1 we see that, if $L u=0$ in $\Omega$, then $\int_{\partial \Omega} \frac{\partial u}{\partial \nu} d \sigma=0$. Denote the space of $L^{p}(\partial \Omega)$ functions with mean value zero by $L_{0}^{p}(\partial \Omega)$.

Theorem 6.3. Let $\Omega \subset \mathbb{C}$ be a $C^{1}$ polygon. Given $L$, let $p_{0}^{\prime}=p_{k, \lambda}$ be as in Lemma 5.1 and $\frac{1}{p_{0}^{\prime}}+\frac{1}{p_{0}}=1$. Then for all $1<p<p_{0}$

(i) $-\frac{1}{2} I+K^{*}: L_{0}^{p}(\partial \Omega) \rightarrow L_{0}^{p}(\partial \Omega)$ is invertible and

(ii) $\frac{1}{2} I+K^{*}: L^{p}(\partial \Omega) \rightarrow L^{p}(\partial \Omega)$ is invertible.

Proof. By the same arguments used in [LP83] or [DL92] the operator $K$ may be written as system of Hardy kernel operators on a finite interval together with compact operators. Further, as in these papers the index of the system together with $\pm \frac{1}{2} I$ will be zero when $\infty>p^{\prime}>p_{0}^{\prime}$. Thus by the Riesz-Schauder theory we need only establish that the range of $\pm \frac{1}{2} I+K$ is dense in $L^{p^{\prime}}$, i.e., that $\pm \frac{1}{2} I+K^{*}$ is injective in $L^{p}$ or $L_{0}^{p}$. But this follows now by potential theoretic arguments as in [FJR78] (see also [Ver84] for two dimensional arguments in the exterior domain $\bar{\Omega}^{c}$ ), since we have Theorems 6.1 and 6.2. In particular, both $\pm \frac{1}{2} I+K^{*}$ are seen to be invertible on $L_{0}^{p}$. Further, if $\left(\frac{1}{2} I+K^{*}\right) f=0$ it follows, since $-\frac{1}{2} I+K^{*}$ maps $L^{p}$ onto $L_{0}^{p}$, that $f \in L_{0}^{p}$. Thus neither $\left(\frac{1}{2} I+K^{*}\right)(1)$ nor $\left(\frac{1}{2} I+K^{*}\right)(i)$ vanish and together with $L_{0}^{p}$ span all of $L^{p}$, thus establishing (ii). 
By Green's formula, $\frac{1}{2} I+K$ maps any constant to itself. Let $L^{p}(\partial \Omega) /\left\langle e^{i \theta}\right\rangle$ denote the quotient space with respect to the constant functions. The next theorem follows by duality.

Theorem 6.4. With the same hypotheses as Theorem 6.3

(i) $-\frac{1}{2} I+K: L^{p^{\prime}}(\partial \Omega) /\left\langle e^{i \theta}\right\rangle \rightarrow L^{p^{\prime}}(\partial \Omega) /\left\langle e^{i \theta}\right\rangle$ is invertible and

(ii) $\frac{1}{2} I+K: L^{p^{\prime}}(\partial \Omega) \rightarrow L^{p^{\prime}}(\partial \Omega)$ is invertible whenever $p_{0}^{\prime}<p^{\prime}<\infty$.

Theorem 6.5 (Neumann Problem). Let $\Omega$ be a $C^{1}$ polygon, and let $p_{0}$ be as in Theorem 6.3. Let $1<p<p_{0}$ and $g \in L_{0}^{p}(\partial \Omega)$ be given.

(i) There exists a unique solution $u$ in $\Omega$ to

(a) $L u=0$,

(b) $\mathcal{N}(|\partial u|+|\bar{\partial} u|) \in L^{p}(\partial \Omega)$,

(c) $\int_{\partial \Omega} u d \sigma=0$,

(d) $\frac{\partial u}{\partial \nu}=g$ a.e. $(d \sigma) ;$

(ii) there exists a unique solution $u$ in $\mathbb{C} \backslash \bar{\Omega}$ to

(a) $L u=0$,

(b) $\mathcal{N}(|\partial u|+|\bar{\partial} u|) \in L^{p^{\prime}}(\partial \Omega)$,

(c) $u(\zeta)=O\left(|\zeta|^{-1}\right)$ as $|\zeta| \rightarrow \infty$,

(d) $\frac{\partial u}{\partial \nu}=g$ a.e. $(d \sigma)$.

Furthermore, in both cases we have the estimate

(iii) $\|\mathcal{N}(|\partial u|+|\bar{\partial} u|)\|_{L^{p}(\partial \Omega)} \leq C_{p, \Omega}\|g\|_{L^{p}(\partial \Omega)}<\infty$.

Proof. Existence, uniqueness and estimate (iii) follow from Theorems 4.4, 6.2 and 6.3 .

Since (iii) depends on the invertibility of operators from Theorem 6.3 and therefore the theory of compact operators, the quantitative dependence of $C_{p, \Omega}$ on the $C^{1}$ domains $\Omega$ is obscure. We also note that although $\frac{1}{2} I+K^{*}$ is invertible on all of $L^{p}(\partial \Omega), \mathcal{S}(1)(\zeta)$ and $\mathcal{S}(i)(\zeta) \neq O\left(|\zeta|^{-1}\right)$ as $|\zeta| \rightarrow \infty$.

To get a uniqueness result for the Dirichlet problem we need to produce a Green operator with the appropriate estimates. To do this we first solve the regularity problem, to which we now turn our attention.

Basing arguments on the same type of spectral analysis of [LP83] that was used in Theorem 6.3, we could show that the single layer potential $\mathcal{S}$ is invertible from $L^{p}(\partial \Omega)$ to $L_{1}^{p}(\partial \Omega)$ (the Sobolev space of $L^{p}$ functions with first derivatives in $L^{p}$ ) by studying $\frac{\partial}{\partial \tau} \mathcal{S} f(P), P \in \partial \Omega$. However, in this case we would not obtain the identity operator plus Hardy kernels plus compact operators. Instead we would get, in addition to the Hardy kernels, operators that were essentially Hilbert transforms. Though this is not a serious problem, we will instead use some potential theoretic techniques, the analogues of [Ver84, §4].

Let $f$ and $g$ denote holomorphic functions. Recall the regular solutions to $L u=0$ of $(12)$ :

$$
u(\zeta)=f((1+k) \zeta-(1-k) \bar{\zeta})+\frac{1+\frac{k}{\lambda}}{1+k} \overline{g(\zeta)}+\frac{1-\frac{k}{\lambda}}{1-k} g(\zeta)
$$


The columns of $\Gamma$ given by (25) and (26) are also seen to be of this form. Thus Theorem 6.5 shows that every solution in a $C^{1}$ polygon is of the form (50). Further, by requiring, as we may, that $g\left(\zeta_{0}\right)=0$ for some fixed $\zeta_{0}$ in the domain, we see that $f$ and $g$ are uniquely determined. We shall assume this normalization in the following.

Definition 1. Given $u$ as in (50) with $g$ normalized, define the conjugate solution to $u$ by

$$
\tilde{u}(\zeta)=-i f((1+k) \zeta-(1-k) \bar{\zeta})+i \frac{1+\frac{k}{\lambda}}{1+k} \overline{g(\zeta)}-i \frac{1-\frac{k}{\lambda}}{1-k} g(\zeta)
$$

For $k=1$ we have $u(\zeta)=f(\zeta)+\left(1+\frac{1}{\lambda}\right) \overline{g(\zeta)}+\left(1-\frac{1}{\lambda}\right) \bar{\zeta} g^{\prime}(\zeta)$ and

$$
\tilde{u}(\zeta)=-i f(\zeta)+i\left(1+\frac{1}{\lambda}\right) \overline{g(\zeta)}-i\left(1-\frac{1}{\lambda}\right) \bar{\zeta} g^{\prime}(\zeta)
$$

We note that $\widetilde{\widetilde{u}}=-u$. One reason for this choice of definition is that we arrive at a rotation-invariant Cauchy-Riemann system, which we state without proof.

Theorem 6.6. Given a solution $u$ and any direction $N \in \mathbb{C}$ with conormal and tangential derivatives defined by (21) and (22), we have

$$
\frac{\partial}{\partial \nu} u=\frac{\partial}{\partial \tau} \tilde{u}
$$

and

$$
\frac{\partial}{\partial \tau} u=-\frac{\partial}{\partial \nu} \tilde{u}
$$

In addition, for $N$ fixed, the conjugate of $\frac{\partial}{\partial \tau} u$ is $\frac{\partial}{\partial \nu} u$, i.e., both are solutions and

$$
\frac{\partial}{\partial \nu} u=\frac{\partial}{\partial \tau} u
$$

Given a solution $u$, Theorem 6.6 provides the standard local algorithm for arriving at $\tilde{u}$, viz. integrating $\frac{\partial}{\partial \nu} u$ in the $\tau$ direction. In light of Theorems 4.4, 6.3 and 6.5 Theorem 6.6 suggests another method that is global in nature. Define the potential

$$
\left(\mathcal{S} \frac{\partial}{\partial \tau}\right) f(\xi)=\int_{\partial \Omega} \frac{\partial \Gamma}{\partial \tau_{\zeta}}(\zeta-\xi) f(\zeta) d \sigma(\zeta) .
$$

Like Theorem 4.3, the next theorem is standard.

Theorem 6.7. With the same hypothesis as Theorem 4.3

(i) $\left\|\mathcal{N}\left(\left(\mathcal{S} \frac{\partial}{\partial \tau}\right) f\right)\right\|_{L^{p}(\partial \Omega)} \leq C_{p}\|f\|_{L^{p}(\partial \Omega)}$,

(ii) $\left(\mathcal{S} \frac{\partial}{\partial \tau}\right) f(\xi) \stackrel{\text { n.t. }}{\rightarrow}$ p.v. $\int_{\partial \Omega} \frac{\partial \Gamma}{\partial \tau_{\zeta}}(\zeta-P) f(\zeta) d \sigma(\zeta)$ a.e. $d \sigma(P), \xi \in \mathbb{C} \backslash \partial \Omega$ and

(iii) the singular integral in (ii) is bounded from $L^{p}$ to $L^{p}, 1<p<\infty$.

By Theorem 6.6 the conjugate of $\left(\mathcal{S} \frac{\partial}{\partial \tau}\right) f$ is the double layer potential $\mathcal{K} f$. This and Green's formula ((iv) of Theorem 4.2) lead to the next result. Denote the Sobolev space of $L_{1}^{p}(\partial \Omega)$ functions with mean value zero by $L_{1,0}^{p}(\partial \Omega)$. 
Theorem 6.8. With the same hypothesis as Theorem 6.3 let $\Omega \subset \mathbb{C}$ be a $C^{1}$ polygon. Then for all $1<p<p_{0}$

(i) $\frac{\partial}{\partial \nu} \mathcal{K}: L_{1,0}^{p}(\partial \Omega) \rightarrow L_{0}^{p}(\partial \Omega)$ is invertible, and

(ii) $\frac{\partial}{\partial \tau} \mathcal{S}: L_{0}^{p}(\partial \Omega) \rightarrow L_{0}^{p}(\partial \Omega)$ is invertible.

Proof. To prove (i) let $u$ be any solution to the Neumann problem. By Theorem $6.5 u \in L_{1}^{p}(\partial \Omega)$, and by Green's formula $u=\mathcal{K} u-\mathcal{S} \frac{\partial u}{\partial \nu}$ in $\Omega$. When we take the conormal derivative in $\Omega$, Green's formula becomes

$$
\left(\frac{1}{2} I+K^{*}\right) \frac{\partial u}{\partial \nu}=\frac{\partial}{\partial \nu} \mathcal{K} u
$$

By (i) and (ii) of Theorem $6.3 \frac{1}{2} I+K^{*}$ is invertible on $L_{0}^{p}$, whence $\frac{\partial}{\partial \nu} \mathcal{K}$ is onto $L_{0}^{p}$ from $L_{1}^{p}$. If $\frac{\partial}{\partial \nu} \mathcal{K} u=0$ a.e. $(d \sigma)$, then, by uniqueness for the Neumann problem, $\mathcal{K} u=$ constant in $\Omega$. Since $u \in L^{p^{\prime}}(\partial \Omega)$ also, (ii) of Theorem 6.4 shows that $u=$ constant on $\partial \Omega$. This proves (i).

To prove (ii) let $f$ be any $L_{1,0}^{p}$ function. By the Cauchy-Riemann equations

$$
\frac{\partial}{\partial \nu} \mathcal{K} f=\frac{\partial}{\partial \nu}\left(\widetilde{\mathcal{S} \frac{\partial}{\partial \tau}}\right) f=-\frac{\partial}{\partial \tau}\left(\mathcal{S} \frac{\partial}{\partial \tau}\right) f=\frac{\partial}{\partial \tau} \mathcal{S}\left(\frac{\partial}{\partial \tau} f\right) .
$$

By (i) therefore $\frac{\partial}{\partial \tau} \mathcal{S}$ maps $L_{0}^{p} 1-1$ onto $L_{0}^{p}$.

The next theorem is proven in a way similar to Theorem 6.5.

Theorem 6.9 (Regularity Problem). Let $\Omega$ be a $C^{1}$ polygon, and let $p_{0}$ be as in Theorem 6.3. Given $1<p<p_{0}$ and $g \in L_{1}^{p}(\partial \Omega)$, there exists a unique solution $u$ to

(i) (a) $L u=0$ in $\Omega$,

(b) $u \stackrel{\text { n.t. }}{\rightarrow}$ g a.e. $(d \sigma)$,

(c) $\mathcal{N}(|\partial u|+|\bar{\partial} u|) \in L^{p}(\partial \Omega)$,

and

(ii) (a) $L u=0$ in $\mathbb{C} \backslash \bar{\Omega}$,

(b) $u \stackrel{\text { n.t. }}{\rightarrow} g$ a.e. $(d \sigma)$,

(c) $\mathcal{N}(|\partial u|+|\bar{\partial} u|) \in L^{p}(\partial \Omega)$,

(d) $(|\partial u|+|\bar{\partial} u|)(\zeta)=O\left(|\zeta|^{-2}\right)$ as $|\zeta| \rightarrow \infty$.

In addition, in both cases we have the estimate

(iii) $\|\mathcal{N}(|\partial u|+|\bar{\partial} u|)\|_{L^{p}(\partial \Omega)} \leq C_{p, \Omega}\left\|\frac{\partial}{\partial \tau} g\right\|_{L^{p}(\partial \Omega)}$.

Proof. By (ii) of Theorem 6.8 there is an $f \in L_{0}^{p}$ so that $\frac{\partial}{\partial \tau} \mathcal{S} f=\frac{\partial g}{\partial \tau}$ a.e. $(d \sigma)$. Thus $u$ may be taken to be $\mathcal{S} f$ plus a constant solution. The rest follows in a manner similar to Theorem 6.5.

Definition 2. Let $H^{\xi}(\zeta)$ denote an operator with columns $H_{1}^{\xi}(\zeta), H_{2}^{\xi}(\zeta)$ that are solutions in $\zeta$ with the same boundary values as $\Gamma_{1}(\zeta-\xi)$ and $\Gamma_{2}(\zeta-\xi)$ respectively. 
Then

$$
\Gamma(\zeta-\xi)-H^{\xi}(\zeta)=G^{\xi}(\zeta)
$$

is the Green operator for $L$.

With an eye toward the study of the Poisson kernel in the next section, we now define tents $T_{\delta}(\xi)$ of slope $\delta$ for $\xi \in \Omega$ close enough to the boundary. The following definition is necessitated because of the technical difficulties encountered on $C^{1}$ domains when estimates such as (iii) from the last theorem are not dilation invariant, the $C_{p, \Omega}$ depending as it does on the non-dilation-invariant properties of $C^{1}$ curves. Let $B_{R}$ denote a ball of radius $R$ centered at the origin. By the definition of Lipschitz domains we may consider a finite number of sets $B_{2 R} \cap \Omega=$ $\{\xi=x+i y \mid y>\phi(x)\} \cap B_{2 R}$, where $\phi: \mathbb{R} \rightarrow \mathbb{R}$ is Lipschitz, and consider points $x_{0}+i y_{0}=\xi_{0} \in B_{R} \cap \Omega$. Let $\chi(x)=0$ for $|x|>1$ and $\chi(x)=|1-x|$ for $|x|<1$. Let $\delta>0$ denote a slope and $t>0$ a scale. Then we define the tent for $\xi_{0}$ with slope $\delta$ by

$$
T_{\delta}\left(\xi_{0}\right)=\left\{x+i y \in \Omega \mid \phi(x)<y<\phi(x)+t \delta \chi\left(\frac{x-x_{0}}{t}\right) \text { with } t \delta=2\left(y_{0}-\phi\left(x_{0}\right)\right)\right\} .
$$

Let $\operatorname{dist}(\xi)$ denote the distance from $\xi$ to $\partial \Omega$. If $\left\|\phi^{\prime}\right\|_{\infty} \leq M$, then, for all $\xi$ close enough to $\partial \Omega$,

$$
\left|\partial T_{\delta}(\xi)\right| \leq C_{M, \delta} \operatorname{dist}(\xi),
$$

and locally $\partial\left(\Omega \backslash T_{\delta}(\xi)\right)$ is given by the graph of a Lipschitz function $\phi_{\xi, \delta}(x)$ so that

$$
\left\|\phi^{\prime}-\phi_{\xi, \delta}^{\prime}\right\| \leq \delta
$$

Let $f_{E}$ denote the integral average with respect to Lebesgue measure, i.e. $\frac{1}{|E|} \int_{E}$. By the last theorem and the explicit boundary values from $\Gamma$ we have the following estimates.

Theorem 6.10. Let $\Omega$ be a $C^{1}$ polygon, let $p_{0}$ be as in Theorem 6.3, and let $G^{\xi}(\zeta)=\Gamma(\zeta-\xi)-H^{\xi}(\zeta)$ be the Green function for $\Omega$ as defined above. Then, for $\xi$ close enough to the boundary and any $1<p<p_{0}$ and any $1 \leq q \leq \infty$,

(i) $\left(\int_{\partial \Omega} \mathcal{N}\left(\left|\partial H^{\xi}\right|+\left|\bar{\partial} H^{\xi}\right|\right)^{p} d \sigma\right)^{1 / p} \leq C_{p, \Omega} \operatorname{dist}(\xi)^{-1 / p^{\prime}}$,

(ii) $\left(f_{\partial T_{\delta}(\xi) \cap \Omega}\left|\partial G^{\xi}\right|^{p}+\left|\bar{\partial} G^{\xi}\right|^{p} d \sigma\right)^{1 / p} \leq C_{\delta, p, \Omega} \frac{1}{\operatorname{dist}(\xi)}$,

(iii) $\left(f_{\partial T_{\delta}(\xi) \cap \Omega}\left|G^{\xi}\right|^{q} d \sigma\right)^{1 / q} \leq C_{\delta, p, \Omega}$.

Here $C_{\delta, p, \Omega}<\infty$ depends on $p, \delta$, the angles of $\Omega$ and the Riesz-Schauder theory of $\Omega$.

Theorem 6.11 (Dirichlet problem in $\Omega$ ). Let $\Omega$ be a $C^{1}$ polygon, and let $p_{0}$ be as in Theorem 6.3. Given $p_{0}^{\prime}<p^{\prime}<\infty$ and $g \in L^{p^{\prime}}$, there exists a unique solution $u$ in $\Omega$ to

(a) $L u=0$,

(b) $\mathcal{N}(u) \in L^{p^{\prime}}(\partial \Omega)$,

(c) $u \stackrel{\text { n.t. }}{\rightarrow}$ g a.e. $(d \sigma)$. 
Furthermore,

(d) $\|\mathcal{N}(u)\|_{L^{p^{\prime}}(\partial \Omega)} \leq C_{p, \Omega}\|g\|_{L^{p^{\prime}}(\partial \Omega)}<\infty$

Proof. Existence follows from Theorems 4.3 and 6.4.

To prove uniqueness, (i) of Theorem 6.10 allows one to argue exactly as in [FJR78]

\section{A WEAK MAXIMUM PRINCIPLE FOR NONSYMMETRIC SYSTEMS}

A weak maximum principle for solutions to symmetric systems and higher order operators in Lipschitz domains in dimensions 2 and 3 has been obtained recently in [DK90], [PV93], [PV95b], [PV95a], [Ver96] and [DK $\left.{ }^{+}\right]$. An important ingredient in the basic proof is the existence of $L^{2}(\partial \Omega)$ estimates, i.e. solvability of the $L^{2}$ Dirichlet and regularity problems in Lipschitz domains. Even when a weak maximum principle or its relatives, Hardy space atomic estimates and BMO estimates, are obtained in $C^{1}$ domains, the $L^{2}$ Lipschitz domain estimates have played a role. See [FK81], [PV93]. The reason for this is that within a given domain subdomains of all scales are constructed for these arguments, and therefore certain estimates must be shown to be scale (dilation) invariant.

For nonsymmetric systems there is as yet no Lipschitz domain theory leading to solvability of boundary value problems. In fact, as demonstrated in $\S 8$, such an $L^{2}$ theory is false for a certain interval of canonical ellipticity $k$. Thus in our setting of $C^{1}$ polygons we encounter two new problems: the lack of $L^{2}$ estimates and the lack of dilation invariant estimates for the $L^{p}$ estimates we do have. As is made clear below, the first problem poses no difficulty, mainly because Sobolev embedding is rather impressive on a 1-dimensional boundary. For the second we make careful use of any dilation invariant estimates we do possess, distinguishing them from the estimates of Theorems $6.11,6.10$ and 6.9. To this end $C$ will denote a constant that depends at most on the Lipschitz nature of $\Omega$ and $1<p<\infty$. $C_{\Omega}$ will denote a constant that depends on less quantifiable properties of $\Omega$, e.g., the action of the compact operators connected with our potentials. In effect $C$ will indicate the use of the dilation invariant theory of Calderón-Zygmund singular integrals [CMM82] while $C_{\Omega}$ will indicate the use of the inverse operators to $\mathrm{C}-\mathrm{Z}$ singular integrals from Theorems $6.3,6.4$ or 6.8 .

The first lemma allows us to pass from the nontangential maximal function to functions defined on the boundary in a dilation invariant manner.

Lemma 7.1. Let $\Omega \subset \mathbb{C}$ be a bounded Lipschitz domain, $1<p<\infty$, Lu $=0$ in $\Omega$ with $\mathcal{N}(|\partial u|+|\bar{\partial} u|) \in L^{p}(\partial \Omega)$, and let $\mathcal{K}$ and $\mathcal{S}$ be defined with respect to $\Omega$.

Let $\frac{\partial}{\partial \tau_{0}}$ denote any fixed directional derivative as in (22) and let $\frac{\partial}{\partial \nu_{0}}$ denote its fixed conormal (21). Then

(i) $\frac{\partial}{\partial \tau_{0}} u(\zeta)=\mathcal{K}\left(\frac{\partial}{\partial \tau_{0}} u\right)(\zeta)+\left(\mathcal{S} \frac{\partial}{\partial \tau}\right)\left(\frac{\partial}{\partial \nu_{0}} u\right)(\zeta)$ for all $\zeta \in \Omega$ and

(ii) $\|\mathcal{N}(|\partial u|+|\bar{\partial} u|)\|_{L^{p}(\partial \Omega)} \leq C|||\partial u|+|\bar{\partial} u| \|_{L^{p}(\partial \Omega)}$.

Proof. Part (i) follows from Green's formula and the Cauchy-Riemann results of Theorem 6.6. Part (ii) follows from (i) and Theorems 4.3, 6.7. 
Let $B_{R}$ denote a ball of radius R. By $\Gamma(w)(\xi)=\int_{\Omega} \Gamma(\xi-\zeta) w(\zeta) d x d y$ we denote the Newtonian potential of $w$.

Lemma 7.2. Let $\Omega \subset \mathbb{C}$ be a bounded Lipschitz domain, $1<p<\infty, k<1$ and $k \leq \lambda<1$. Suppose $w$ is supported in $\Omega \cap B_{R}$. Then

$$
\left\|\frac{\partial}{\partial \nu} \Gamma(w)\right\|_{L^{p}(\partial \Omega)} \leq C \frac{\frac{\lambda}{k}-k}{1-\lambda} R^{\frac{1}{p^{\prime}}}\|w\|_{L^{p}(\Omega)}
$$

Proof. The left side is equal to

$$
\begin{aligned}
& \sup \operatorname{su}_{(M-k \widetilde{M}) f \|_{L^{p^{\prime}}(\partial \Omega)}=1} \Re \int_{\partial \Omega}(M-k \widetilde{M}) f \frac{\partial}{\partial \nu} \Gamma(w) d \sigma \\
= & \sup _{f} \Re \int_{\Omega}(M-k \widetilde{M}) \mathcal{K} f w d x d y \\
\leq & \sup _{f} 2\left(\frac{\lambda}{k}-k\right)\|\mathcal{K} f\|_{L^{p^{\prime}}\left(B_{R}\right)}\|w\|_{L^{p}\left(B_{R}\right)} \\
\leq & \sup _{f} 2\left(\frac{\lambda}{k}-k\right) R^{\frac{1}{p^{\prime}}}\|\mathcal{N}(\mathcal{K} f)\|_{L^{p^{\prime}}(\partial \Omega)}\|w\|_{L^{p}\left(B_{R}\right)} \\
\leq & \sup _{f} 2\left(\frac{\lambda}{k}-k\right) R^{\frac{1}{p^{\prime}}} C\|f\|_{L^{p}(\partial \Omega)}\|w\|_{L^{p}(\Omega)} \\
\leq & \frac{\frac{\lambda}{k}-k}{1-\lambda} R^{\frac{1}{p^{\prime}} C\|w\|_{L^{p}(\Omega)} .}
\end{aligned}
$$

Here the equality is Fubini combined with the Hermitian property of $(M-k \widetilde{M}) \mathcal{K}$ as in (34), and the second inequality from the end is (i) of Theorem 4.3.

Lemma 7.3. Let $\Omega \subset \mathbb{C}$ be a $C^{1}$ polygon; $L$ and $p_{0}$ as in Theorem 6.3. Suppose

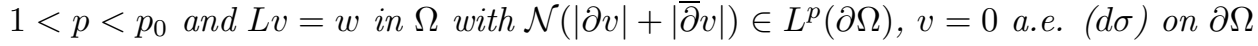
and $v$ supported in $B_{R} \cap \Omega$. Then

$$
\left(f_{B_{R} \cap \partial \Omega}\left|\frac{\partial v}{\partial \nu}\right|^{p} d \sigma\right)^{\frac{1}{p}} \leq R C_{\Omega}\left(f_{B_{R} \cap \Omega}|w|^{p} d x d y\right)^{\frac{1}{p}}
$$

Proof. By Green's formula $v=-\mathcal{S} \frac{\partial v}{\partial \nu}+\Gamma(w)$, whence by (ii) of Theorem 4.4 $\left(\frac{1}{2} I+K^{*}\right) \frac{\partial v}{\partial \nu}=\frac{\partial}{\partial \nu} \Gamma(w)$. By Theorem 6.3 the left side of (53) is bounded by $C_{\Omega} R^{-\frac{1}{p}}\left(\int_{\partial \Omega}\left|\frac{\partial}{\partial \nu} \Gamma(w)\right|^{p} d \sigma\right)^{\frac{1}{p}}$. For nonsymmetric systems (53) follows by Lemma 7.2 .

For convenience we will use the notation $|\nabla u|$ in place of $|\partial u|+|\bar{\partial} u|$.

Lemma 7.4. With the hypotheses of Lemma 7.3 suppose $u$ is defined in $\Omega$ with $\mathcal{N}(\nabla u) \in L^{p}(\partial \Omega), L u=0$ in $B_{4 R} \cap \Omega$ and $u=0$ a.e. $(d \sigma)$ on $B_{4 R} \cap \partial \Omega$. Define the truncated maximal function $\mathcal{N}_{1}(\nabla u)(P)=\sup _{\zeta}|\nabla u(\zeta)|$, where the sup is taken over the nontangential approach region (33) intersected with $B_{R}$. Then

$$
\left(f_{B_{R} \cap \partial \Omega} \mathcal{N}_{1}(\nabla u)^{p} d \sigma\right)^{\frac{1}{p}} \leq C C_{\Omega}\left(f_{B_{4 R}}|\nabla u|^{p} d x d y\right)^{\frac{1}{p}} .
$$


Proof. By Lemma 7.1 the left side is bounded by $C\left(f_{\partial\left(B_{2 R} \cap \Omega\right)}|\nabla u|^{p} d \sigma\right)^{\frac{1}{p}}$. Let $\chi$ be a smooth real-valued cut-off function identically equal to 1 on $B_{2 R}$ and supported in $B_{3 R}$, so that first and second derivatives of $\chi$ are no larger than $C R^{-1}$ and $C R^{-2}$ respectively. Let $v=\chi u$. Then $|L v| \leq C R^{-1}|\nabla u|+C R^{-2}|u|$. Since $u$ vanishes on $B_{2 R} \cap \partial \Omega$, Lemma 7.3 implies

$$
\begin{aligned}
\left(f_{B_{2 R} \cap \partial \Omega}|\nabla u|^{p} d \sigma\right)^{\frac{1}{p}} & \leq C_{\Omega}\left(f_{B_{3 R} \cap \Omega}|\nabla u|^{p} d x d y\right)^{\frac{1}{p}} \\
& +R^{-1} C_{\Omega}\left(f_{B_{3 R} \cap \Omega}|u|^{p} d x d y\right)^{\frac{1}{p}} .
\end{aligned}
$$

The Poincaré inequality may be used to control the last term. The resulting inequality may be established for $R$ replaced by $\tilde{R}$, where $R \leq \tilde{R} \leq \frac{4}{3} R$, and the resulting inequalities averaged in $\tilde{R}$ so that the $\left(f_{\Omega \cap \partial B_{2 \tilde{R}}}|\nabla u|^{p} d \sigma\right)^{\frac{1}{p}}$ terms may also be replaced with the solid integral on the right of (54).

With the last lemma we are set to exploit the argument of Dahlberg and Kenig [DK90] for solutions to the regularity problem with atomic data on $\partial \Omega$. Since the Green operator vanishes on $\partial \Omega$, (ii) of Theorem 6.10 shows that $\frac{\partial}{\partial \tau_{P}} G^{\xi}(P)$ for $P \in \partial(\Omega \backslash T(\xi))$ is a 1-p-atom [CW77], $1<p<p_{0}$, on the boundary of the $C^{1}$ polygons $\Omega \backslash T_{\delta}(\xi)$. Our intention, as in [PV93] and [DK90], is to obtain the regularity atomic estimate for the Green function on these domains and thus establish the proper estimates on the Poisson kernel for the operators $L$ in $\Omega$, from which the weak maximum principle will follow. But to do this, once given a $C^{1}$ polygon $\Omega$, we need to be sure that the regularity and Dirichlet estimates of Theorems 6.9 and 6.11 may be applied uniformly over all the domains $\Omega \backslash T_{\delta}(\xi)$ for some fixed $\delta>0$ with a constant $C_{\Omega}<\infty$ independent of $\xi$. Thus the need for the property (52) of our tents $T_{\delta}$.

Lemma 7.5. Let $\Omega \subset \mathbb{C}$ be a $C^{1}$ polygon, and let $p_{0}$ be as in Theorem 6.3. For $\operatorname{dist}(\xi)$ small enough let $\Omega_{\xi, \delta}=\Omega \backslash T_{\delta}(\xi)$. Then there exist a $\delta>0$ and a constant $C_{\Omega}=C_{\Omega, p, \delta}<\infty$ independent of $\xi$ so that if $u$ is a solution to $L u=0$ in $\Omega_{\xi, \delta}$, then,

$$
\text { (i) }\|\mathcal{N}(u)\|_{L^{p^{\prime}}\left(\partial \Omega_{\xi, \delta}\right)} \leq C_{\Omega}\|u\|_{L^{p^{\prime}}\left(\partial \Omega_{\xi, \delta}\right)} \text { if } \mathcal{N}(u) \in L^{p^{\prime}}\left(\partial \Omega_{\xi, \delta}\right),
$$

and

(ii) $\|\mathcal{N}(\nabla u)\|_{L^{p}\left(\partial \Omega_{\xi, \delta}\right)} \leq C_{\Omega}\left\|\frac{\partial}{\partial \tau} u\right\|_{L^{p}\left(\partial \Omega_{\xi, \delta}\right)}$ if $\mathcal{N}(\nabla u) \in L^{p}\left(\partial \Omega_{\xi, \delta}\right)$.

Proof. The estimates of Theorems 6.9 and 6.11 come from the boundedness of the operators inverse to the singular integral operators of Theorems 6.4 and 6.8 . Since the set of bounded linear operators with bounded inverses is open in the operator topology, it suffices to have the singular integrals on the $\partial \Omega_{\xi, \delta}$ close enough in operator norm to those of $\partial \Omega$ for the norms of the inverses to be uniformly controlled. By [CMM82], particularly Theorem XI, and other arguments in [FJR78] (see [Ver87, pp 872-873] for an example of the type of argument used), our singular integral operators are a continuous (but nonlinear) mapping from the space of Lipschitz functions under uniform convergence of the gradient to the bounded linear 
operators. Thus the existence of the constant $C_{\Omega}<\infty$ follows by taking $\delta>0$ small enough in (52).

Theorem 7.1 (Regularity atomic estimate). Let $\Omega \subset \mathbb{C}$ be a $C^{1}$ polygon, and let $p_{0}$ be as in Theorem 6.3. Let $1<p<p_{0}$, and suppose $u$ is a solution in $\Omega$ to the regularity problem (Theorem 6.9) with $L_{1}^{p}(\partial \Omega)$ data $g$. Suppose $g$ is supported in $\Delta_{r}=B_{r} \cap \partial \Omega$ with $B_{r}$ centered at a boundary point, which we may take to be the origin in $\mathbb{C}$. Then if $\left(f_{\Delta_{r}}\left|\frac{\partial}{\partial \tau} g\right|^{p} d \sigma\right)^{\frac{1}{p}}=\frac{1}{\left|\Delta_{r}\right|}$ it follows that

$$
\|\mathcal{N}(\nabla u)\|_{L^{1}(\partial \Omega)} \leq C_{\Omega, p}
$$

where the constant is independent of $r$ and $g$.

Proof. By rescaling we take $r=1$, while the regularity and Dirichlet estimates of Theorems 6.9 and 6.11 remain unchanged. As usual, the local estimate

$$
\int_{\Delta_{10}} \mathcal{N}(\nabla u) d \sigma \leq C_{\Omega, p}
$$

follows by Hölder's inequality and the $L^{p}$ estimate of Theorem 6.9.

Next let $\alpha$ be the parameter in the nontangential approach regions (33) for $\Omega$ and define

$$
\Omega_{2}=\bigcup_{P \in \Delta_{5}}\{\zeta \in \Omega:|\zeta-P| \leq(1+10 \alpha) \operatorname{dist}(\zeta)\}
$$

For each $P \in \partial \Omega \backslash \Delta_{10}$ define an interior maximal function

$$
\mathcal{N}_{2}(\nabla u)(P)=\sup \left\{|\nabla u(\zeta)|: \zeta \in \Omega_{2} \cap\{|\zeta-P| \leq(1+\alpha) \operatorname{dist}(\zeta)\}\right\}
$$

and the truncated nontangential maximal function $\mathcal{N}_{1}(\nabla u)(P)$ as in $(55)$ with $\Omega_{2}$ replaced by $\Omega \backslash \Omega_{2}$.

$\mathcal{N}_{2}(\nabla u)(P)$ is easily controlled for $P \in \partial \Omega \backslash \Delta_{10}$ by observing that for any $\zeta$ in the regions $(55), \operatorname{dist}(\zeta) \geq C_{\alpha}|P|$. By interior estimates (e.g., [Joh55] or (i) of Lemma 7.1 may be used to produce mean value theorems $)|\nabla u(\zeta)| \leq C \frac{1}{|P|}(f \mathcal{N}(u) d \sigma)$, where the average is over a surface ball of radius comparable to $|P|$. By Hölder's inequality and the $L^{q}$-Dirichlet estimate of Theorem $6.11, p_{0}^{\prime}<q<\infty$, we get

$$
\begin{aligned}
\mathcal{N}_{2}(\nabla u)(P) & \leq C|P|^{-1-\frac{1}{q}}\left(\int_{\partial \Omega}|g|^{q} d \sigma\right)^{\frac{1}{q}} \\
& \leq C|P|^{-1-\frac{1}{q}}
\end{aligned}
$$

and $\mathcal{N}_{2}(\nabla u) \in L^{1}(\partial \Omega)$. For $\mathcal{N}_{1}(\nabla u)$ we consider portions of the boundary $B_{R} \cap \partial \Omega$ as in Lemma 7.4 so that the distance of $B_{R} \cap \partial \Omega$ to the origin is comparable to $R$, and carry out the idea of [DK90] as follows. By Hölder's inequality and Lemma 7.4

$$
\int_{B_{R} \cap \partial \Omega} \mathcal{N}_{1}(\nabla u) d \sigma \leq R C_{\Omega}\left(f_{B_{4 R} \cap \Omega}|\nabla u|^{p}\right)^{\frac{1}{p}} \leq R C_{\Omega}\left(f_{B_{4 R} \cap \Omega}|\nabla u|^{2}\right)^{\frac{1}{2}} .
$$

For nonsymmetric systems the last integral may be dominated by the quadratic form on the right side of (47) of Theorem 6.1. Now Theorem 6.1 is applied to $u$ 
times a smooth cut-off function with $\Omega$ replaced by $B_{5 R} \cap \Omega$ to obtain the Cacciopoli estimate

$$
\begin{aligned}
\left(f_{B_{4 R} \cap \Omega}|\nabla u|^{2}\right)^{\frac{1}{2}} & \leq C R^{-\frac{1}{2}}\left(f_{B_{5 R} \cap \Omega}|u||\nabla u|\right)^{\frac{1}{2}} \\
& \leq C R^{-\frac{1}{2}}\left(f_{B_{5 R} \cap \Omega}|u|^{p^{\prime}}\right)^{\frac{1}{2 p^{\prime}}}\left(f_{B_{5 R} \cap \Omega}|\nabla u|^{p}\right)^{\frac{1}{2 p}} \\
& \leq C R^{-\frac{1}{2}}\left(f_{B_{5 R} \cap \partial \Omega} \mathcal{N}(u)^{p^{\prime}} d \sigma\right)^{\frac{1}{2 p^{\prime}}}\left(f_{B_{5 R} \cap \Omega}|\nabla u|^{p}\right)^{\frac{1}{2 p}} \\
& \leq C_{\Omega} R^{-\frac{1}{2}-\frac{1}{2 p^{\prime}}}\left(f_{B_{5 R} \cap \Omega}|\nabla u|^{p}\right)^{\frac{1}{2 p}}
\end{aligned}
$$

where Theorem 6.11 is used in the last inequality. Thus by (56)

$$
R\left(f_{B_{4 R} \cap \Omega}|\nabla u|^{p}\right)^{\frac{1}{p}} \leq C_{\Omega} R^{-\frac{1}{2 p^{\prime}}} \sqrt{R\left(f_{B_{5 R} \cap \Omega}|\nabla u|^{p}\right)^{\frac{1}{p}}} .
$$

Now let $R=R_{n}=(1+\beta)^{n}$ for some $\beta>0$ and $n=0,1,2, \ldots$ Then $\Omega \backslash \Delta_{10}$ may be covered by the union of the $B_{R_{n}} \cap \partial \Omega$ and, summing (57) over $n$, each of the square roots may be hidden by a finite number of the terms on the left (see [PV93], for example). Thus $\sum_{n} R_{n}\left(f_{B_{4 R_{n}} \cap \Omega}|\nabla u|^{p}\right)^{\frac{1}{p}} \leq C_{\Omega} \sum_{n} R_{n}^{-\frac{1}{p^{\prime}}}$ and, by (56),

$$
\int_{\partial \Omega \backslash \Delta_{10}} \mathcal{N}_{1}(\nabla u) d \sigma \leq C_{\Omega} \sum_{n} R_{n}^{-\frac{1}{p^{\prime}}} \leq C_{\Omega, p}
$$

Corollary 1 (Neumann atomic estimate). If $u$ is a solution to the Neumann problem in $\Omega$ with $L_{0}^{p}(\partial \Omega)$ data $g$ and $g$ is supported in $\Delta_{r}$ with $\left(f_{\Delta_{r}}|g|^{p} d \sigma\right)^{\frac{1}{p}}=\frac{1}{\left|\Delta_{r}\right|}$, then

$$
\|\mathcal{N}(\nabla u)\|_{L^{1}(\partial \Omega)} \leq C_{\Omega, p}
$$

Proof. The proof follows by applying Theorem 6.6 (the Cauchy-Riemann equations) to the last theorem.

We note that for solutions in $\Omega, \frac{\partial u}{\partial \tau}$ and $\frac{\partial u}{\partial \nu}$ always have the mean value zero property required by the definition of $H^{1}$ Hardy space atoms. Recall that the atomic Hardy space $H_{a t}^{1}(\partial \Omega)$ is a Banach space under the norm $\|f\|_{H_{a t}^{1}}=\inf \left\{\sum_{j}\left|\lambda_{j}\right|: f=\right.$ $\sum_{j} \lambda_{j} a_{j}$; the $a_{j}$ are atoms $\}$.

Theorem 7.2 (Regularity and Neumann in $\Omega$ ). With the hypothesis of Theorem 7.1, and $g \in H_{a t}^{1}(\partial \Omega)$ there exists a unique solution $u$ in $\Omega$ to
(a) $L u=0$,
(b) $\mathcal{N}(\nabla u) \in L^{1}(\partial \Omega)$,
(c) $\int_{\partial \Omega} u d \sigma=0$,
(i) $\frac{\partial u}{\partial \tau}=g$ a.e. $(d \sigma)$ (regularity)

and 
(ii) $\frac{\partial u}{\partial \nu}=g$ a.e. $(d \sigma)$ (Neumann).

Furthermore, in each case we have the estimate

$$
\|\mathcal{N}(\nabla u)\|_{L^{1}(\partial \Omega)} \leq C_{\Omega}\|g\|_{H_{a t}^{1}(\partial \Omega)} .
$$

Proof. Existence and uniqueness follow from Theorem 7.1, Theorem 6.2 and Theorem 6.6.

Remark 9. Atomic estimates for solutions in the exterior of $\Omega$ may also be done. Only minor modifications of the proofs of Lemmas 7.3, 7.4 and Theorem 7.1 are needed. For example, in Lemma 7.3 we would obtain $\left(\frac{1}{2} I-K^{*}\right) \frac{\partial v}{\partial \nu}=-\frac{\partial}{\partial \nu} \Gamma(w)$, and the mean value $f_{\partial \Omega} \frac{\partial v}{\partial \nu}$ would have to be subtracted from $\frac{\partial v}{\partial \nu}$ in order to use the invertibility of Theorem 6.3. But by the Gauss divergence theorem $f_{\partial \Omega} \frac{\partial v}{\partial \nu} d \sigma=$ $\frac{1}{|\partial \Omega|} \int_{B_{R} \cap \Omega} w d x d y$, which, when it occurs, gives us the right side of (53) since $1<R<|\partial \Omega|$. Consequently Theorem 7.2 is valid for $\mathbb{C} \backslash \bar{\Omega}$ replacing $\Omega$ and the condition

$$
\left(\mathrm{c}^{\prime}\right) \quad|\nabla u(\zeta)|=O\left(|\zeta|^{-2}\right) \text { as }|\zeta| \rightarrow \infty
$$

replacing (c).

As in [DK90] and [PV93], we now want the estimates of Theorem 7.2 to apply to the gradient of the Green operator (51) and thus yield estimates on the Poisson kernel for nonsymmetric systems that will yield a weak maximum principle $\left(L^{\infty}\right.$ estimates). To do this we will use Lemma 7.5.

Let $\Omega$ be a $C^{1}$ polygon and $u$ a solution to the Dirichlet problem of Theorem 6.11 in $\Omega$ for some $q, p_{0}^{\prime}<q<\infty$. By integration by parts (Theorem 4.1) and (i), (ii) and (iii) of Theorem 4.2 (Green's formula) we obtain for nonsymmetric systems

$$
u(\xi)=\frac{1}{\left(\frac{\lambda}{k}-k\right)(1-\lambda)}(\widetilde{M}-k M) \int_{\partial \Omega}{\overline{\frac{\partial}{\partial \nu_{\zeta}} G \xi(\zeta)}}^{T}(M-k \widetilde{M}) u(\zeta) d \sigma(\zeta) .
$$

Unfortunately it is not clear that a formula like (30) is true for $\frac{\partial}{\partial \nu} G$, so we will be content with (59) for our Poisson representation.

Theorem 7.3 (Weak maximum principle). Let $\Omega \subset \mathbb{C}$ be a $C^{1}$ polygon. Let $L u=$ 0 in $\Omega$ and let $p_{0}$ be as in Theorem 6.3. Then if

(i) $\mathcal{N}(u) \in L^{q}(\partial \Omega)$ for some $p_{0}^{\prime}<q<\infty$

and

(ii) $u \stackrel{\text { n.t. }}{\rightarrow} g \in L^{\infty}(\partial \Omega)$ a.e. $(d \sigma)$,

it follows that

$$
\sup _{\zeta \in \Omega}|u(\zeta)| \leq C_{\Omega}\|g\|_{L^{\infty}(\partial \Omega)},
$$

where $C_{\Omega}$ is independent of $g$ and $q$. 
Proof. We will prove (60) for nonsymmetric systems. For dist $(\xi)$ small enough let $T_{\delta}(\xi)$ be the tents of Lemma 7.5 (i.e., $\delta$ is fixed). Then the integration in the Poisson representation (59) splits into a piece over $I=\partial T_{\delta}(\xi) \cap \partial \Omega$ and one over $I I=\partial \Omega \backslash \partial T_{\delta}(\xi)$. By Hölder's inequality, (i) of Theorem 6.10 and the explicit formula for $\frac{\partial \Gamma}{\partial \nu}$,

$$
\begin{aligned}
\left|\int_{I}{\overline{\frac{\partial}{\partial \nu} G^{\xi}}}^{T}(M-k \widetilde{M}) u d \sigma\right| & \leq C_{p, \Omega}\|(M-k \widetilde{M}) u\|_{L^{\infty}(\partial \Omega)} \\
& \leq\left(\frac{\lambda}{k}-k\right) C_{p, \Omega}\|u\|_{L^{\infty}(\partial \Omega)}
\end{aligned}
$$

for any $1<p<p_{0}$.

Next, each column of $G^{\xi}$ is a solution in the domain $\Omega_{\xi, \delta}$ to the regularity problem with data $\frac{\partial}{\partial \tau} G^{\xi}$ supported on $\partial T_{\delta}(\xi) \cap \Omega$ with the estimate (ii) of Theorem 6.10. Thus by the atomic estimate (Theorem 7.1)

$$
\left|\int_{I I} \overline{\frac{\partial}{\partial \nu}}^{T} G^{T}(M-k \widetilde{M}) u d \sigma\right| \leq C_{\Omega_{\xi, \delta}}\left(\frac{\lambda}{k}-k\right)\|u\|_{L^{\infty}(\partial \Omega)} .
$$

But by Lemma 7.5 applied to the proof of Theorem 7.1 we have that the constant in (61) is in fact independent of $\xi$. Thus, for $\operatorname{dist}(\xi)$ small enough,

$$
|u(\xi)| \leq \frac{\frac{\lambda}{k}-k}{1-\lambda} C_{\Omega}\|u\|_{L^{\infty}(\partial \Omega)} .
$$

Suppose now that $(60)$ has been established for all $\xi$ such that $0<\operatorname{dist}(\xi) \leq \gamma$. One can easily construct a $C^{\infty}$ domain $\Omega^{\prime}$ so that $\partial \Omega^{\prime} \subset \Omega$ and $\operatorname{dist}\left(\partial \Omega^{\prime}, \partial \Omega\right) \leq \gamma / 2$. With respect to $\Omega^{\prime}, \mathcal{N}(u)$ is in every $L^{p}\left(\partial \Omega^{\prime}\right)$. So for $\operatorname{dist}(\xi)>\gamma$, using the geometry of nontangential approach regions,

$$
\begin{aligned}
|u(\xi)| & \leq C \frac{1}{\operatorname{dist}(\xi)^{\frac{1}{p}}}\left(\int_{\partial \Omega^{\prime}} \mathcal{N}(u)^{p} d \sigma^{\prime}\right)^{\frac{1}{p}} \\
& \leq C \frac{1}{\gamma^{\frac{1}{p}}}\left(\int_{\partial \Omega^{\prime}}|u|^{p} d \sigma^{\prime}\right)^{\frac{1}{p}} \leq C \frac{\frac{\lambda}{k}-k}{1-\lambda} \frac{1}{\gamma^{\frac{1}{p}}} C_{\Omega}\|u\|_{L^{\infty}(\partial \Omega)}
\end{aligned}
$$

by (62).

Remark 10. Because Rellich formulas are true for symmetrizable systems $(\lambda>1)$, a dilation invariant $L^{2}(\partial \Omega)$ theory is true for them. Consequently Theorem 7.3 is true in Lipschitz domains for such systems. See [PV95a], [Ver96], [DK ${ }^{+}$.

Remark 11. From Theorem 7.3 the solvability of the Dirichlet problem with continuous data also follows. See [PV93], [PV95b]. Thus we have

Theorem 7.4. With the hypothesis of Theorem 7.3, given $g \in C(\partial \Omega)$ there exists a unique solution $u \in C(\bar{\Omega})$ to $L u=0$ in $\Omega$ so that $u=g$ on $\partial \Omega$.

Remark 12. The opposite inequality to (58) may also be established for solutions to our systems in $C^{1}$ polygons by extending the theorem of Varopoulos [Var77] exactly as in [FK81]. As in the latter paper and [DK87, p. 463], this then leads to invertibility on $H_{a t}^{1}(\partial \Omega)$ of $\frac{1}{2} I-K^{*}$ and invertibility on $B M O(\partial \Omega)$ of $\frac{1}{2} I+K$. Further consequences, such as that $|\nabla u(x, y)|^{2} \operatorname{dist}(x, y) d x d y$ is a Carleson measure on $C^{1}$ polygons when $u$ is a solution to the Dirichlet problem with $B M O$ data, then easily follow by exploiting the potential representations. 


\section{EXAMPLES IN CORNERS}

We show that counterexample functions may be constructed for any of our systems if the equation (64) below holds. For the skewsymmetric systems $(\lambda=k)$ we show that there are counterexamples for any $p, 1 \leq p<\infty$, to the unique solvability of the Dirichlet problem in bounded Lipschitz domains (in fact in a truncated sector). The method is from [CD93]. We point out that the calculations here arrive at some of the same conclusions as the symbol calculation of $\S 5$. We also show that for the skewsymmetric systems we have no $L^{2}$ counterexamples in any sector if we choose $k$ with $1>k>k_{0}$, where $k_{0}$ is positive and not larger than .519 .

Recall the sectors $\Omega_{\alpha, \beta}$ from $\S 5$. Following [CD93] we write the system as a matrix of second order operators

$$
\left[\begin{array}{ll}
\frac{\partial^{2}}{\partial x^{2}}+\lambda \frac{\partial^{2}}{\partial y^{2}} & \frac{\lambda-k^{2}}{k} \frac{\partial^{2}}{\partial y \partial x} \\
(\lambda-1) \frac{\partial^{2}}{\partial y \partial x} & \frac{\lambda}{k} \frac{\partial^{2}}{\partial x^{2}}+k \frac{\partial^{2}}{\partial y^{2}}
\end{array}\right]
$$

and consider solutions (in the variable $\zeta$ ) in a sector $\Omega_{\alpha, \beta}$ of the form

$$
(\mu \zeta+\bar{\zeta})^{z}\left[\begin{array}{l}
q_{1} \\
q_{2}
\end{array}\right]
$$

for $q_{1}, q_{2} \in \mathbb{C}$. For $k \neq 1, \lambda \neq k^{2}$ we find the four solutions

$$
\bar{\zeta}^{z}\left[\begin{array}{l}
i \\
a
\end{array}\right], \zeta^{z}\left[\begin{array}{c}
-i \\
a
\end{array}\right],((1+k) \zeta-(1-k) \bar{\zeta})^{z}\left[\begin{array}{l}
i \\
1
\end{array}\right],(\overline{(1+k) \zeta-(1-k) \bar{\zeta}})^{z}\left[\begin{array}{c}
-i \\
1
\end{array}\right]
$$

with $a=\frac{k(1-\lambda)}{\lambda-k^{2}}$.

Set $\gamma=e^{-2 \pi i z}$ and $w=w(\zeta)=(1+k) \zeta-(1-k) \bar{\zeta}$. Recall that $\beta / \alpha=e^{i \theta}$ and that $2 B=w(\beta), 2 A=w(\alpha)$ (see $\S 5$ ). Using the branch of the logarithm with $0 \leq \arg <2 \pi$ and the four solutions above, we form two solutions which are 0 as $\arg (\zeta) \downarrow \arg (\alpha)$ :

$$
\left[\begin{array}{l}
i\left(\gamma\left(\frac{\bar{\zeta}}{\alpha}\right)^{z}-\left(\frac{\zeta}{\alpha}\right)^{z}\right)-a i\left(\left(\frac{w}{2 A}\right)^{z}-\gamma\left(\frac{\bar{w}}{2 A}\right)^{z}\right) \\
a\left(\gamma\left(\frac{\bar{\zeta}}{\bar{\alpha}}\right)^{z}+\left(\frac{\zeta}{\alpha}\right)^{z}\right)-a\left(\left(\frac{w}{2 A}\right)^{z}+\gamma\left(\frac{\bar{w}}{2 A}\right)^{z}\right)
\end{array}\right]
$$

and

$$
\left[\begin{array}{l}
i\left(\gamma\left(\frac{\bar{\zeta}}{\alpha}\right)^{z}+\left(\frac{\zeta}{\alpha}\right)^{z}\right)-i\left(\left(\frac{w}{2 A}\right)^{z}+\gamma\left(\frac{\bar{w}}{2 A}\right)^{z}\right) \\
a\left(\gamma\left(\frac{\bar{\zeta}}{\alpha}\right)^{z}-\left(\frac{\zeta}{\alpha}\right)^{z}\right)-\left(\left(\frac{w}{2 A}\right)^{z}-\gamma\left(\frac{\bar{w}}{2 A}\right)^{z}\right)
\end{array}\right] .
$$

To obtain a solution which is also 0 on $\zeta=r \beta, r>0$, we need the determinant of the matrix formed from these two solutions to be 0 ,

$$
a\left(\left|\frac{B}{A}\right|^{z}+1\right)+\left|\frac{B}{A}\right|^{z}\left(\left(1+a^{2}\right) \sin z \theta \sin z \psi-2 a \cos z \theta \cos z \psi\right)=0,
$$

where $\psi=\arg \left(\frac{B}{A}\right)$. Recall that $\Lambda=\frac{(1+k)(k-\lambda)}{(1-k)(k+\lambda)}$. Using trigonometric identities the previous equation simplifies to

$$
\left(\Lambda^{2}-1\right)\left(\left|\frac{B}{A}\right|^{z}+\left|\frac{B}{A}\right|^{-z}\right)+2 \cos z(\theta+\psi)-2 \Lambda^{2} \cos z(\theta-\psi)=0 .
$$


Considering $z$ as an indeterminate, not affected by conjugation, we obtain one of the factors in (40) of the symbol calculation:

$$
\Lambda^{2}\left|\sin \frac{z}{2}\left(\theta+i \log \left(\frac{B}{A}\right)\right)\right|^{2}-\left|\sin \frac{z}{2}\left(\theta-i \log \left(\frac{B}{A}\right)\right)\right|^{2}=0
$$

We point out this is also the first factor of equation 4.8 in [DL92, Theorem 4.1] and that the left hand side is an analytic function of $z$.

For the skewsymmetric examples let $\lambda=k<1$, so that $\Lambda=0$, and let $\alpha=1$ and $\beta=i$, so that $\theta=\psi=\pi / 2$ and $\left|\frac{B}{A}\right|=1 / k$. As in the symbol calculation, we find the real part of a zero to be

$$
\Re z=\frac{n \pi \frac{\theta+\psi}{2}}{\left(\frac{\theta+\psi}{2}\right)^{2}+\left(\frac{1}{2} \log \left|\frac{B}{A}\right|\right)^{2}} .
$$

Set $n=-1$. For any $p_{0}>1$ choose $\lambda=k=e^{-\pi \sqrt{2 p_{0}-1}}$. Then, from the discussion above, there is a solution $u(\zeta)$ in the first quadrant with zero boundary values. The power is given by $z_{p_{0}}=-\frac{1}{p_{0}}-i \frac{\sqrt{2 p_{0}-1}}{p_{0}}$. The behavior of $u$ near the origin is of the order $|\zeta|^{\frac{-1}{p_{0}}}$. The nontangential maximal function of $u$ is not in $L^{p_{0}}\left(\partial \Omega_{\alpha, \beta}\right)$; however, $\mathcal{N}(u) \in L^{p}\left(\partial \Omega_{\alpha, \beta}\right)$ for all $p<p_{0}$. Let $D$ be the quarter of the unit disk in the first quadrant and $q$ be large enough (see Theorem 4.15) so that we may uniquely solve the equation in $D$ with boundary values $u$. Let this solution be $w(\zeta)$; then $w-u$ is a nonzero solution with zero boundary values and $\mathcal{N}(w-u) \in L^{p}(\partial D)$ for all $p<p_{0}$. We have proven

Theorem 8.1. For any $1 \leq p<\infty$ there is a strongly elliptic system and a bounded Lipschitz domain for which uniqueness of the solution to the Dirichlet problem fails.

Remark 13. When nonuniqueness occurs in the corners of polygonal domains for $p$ below the critical $p_{0}$ it is possible to recover uniqueness and estimates in $L^{p}(\partial \Omega)$ by modifying the $L^{q}(\partial \Omega)$ solutions, $p_{0}<q$, through the device of adding on a certain multiple of the $p_{0}$ counterexample function. (This is in analogue to the change in the inverse operators to the double layer potential across the critical $p_{0}$ observed in [FJL76].) It is then easy to show that estimates and existence fail for an infinite dimensional set of $L^{p_{0}}$ data. Thus the Dirichlet problem is in fact not Fredholm at the critical $p_{0}$, and is Fredholm both above and below $p_{0}$. Compare with Remark 1.

We now turn our attention to showing

Theorem 8.2. For skewsymmetric systems there is a range of canonical ellipticity, $k_{0}<k<1$, for which there are no $L^{2}$ counterexamples to the unique solvability of the Dirichlet problem in $C^{1}$ polygons.

Remark 14. Thus we have a collection of systems for which we are unable to prove or disprove the uniqueness of solutions to the $L^{2}$ Dirichlet problem in Lipschitz domains.

Proof. We shall prove the positive result: For skewsymmetric systems the $L^{2}$ Dirichlet problem has a unique solution in $C^{1}$ polygons for $k$ near 1 . The idea is to prove 
a suitable replacement of Lemma 5.1 and then apply the analysis in $\S 6$ to obtain uniqueness. We need only show that $|\Re z|>1 / 2$ (see (40) and (42)) for $k$ near 1. By completing the square in (65) we get the desired inequality:

$$
\begin{aligned}
& 4 \theta(2 \pi-\theta)+2(\psi-\theta)(2 \pi-\theta)-2(\psi-\theta) \theta \\
& >(\psi-\theta)^{2}+\left(\log \left|\frac{B}{A}\right|\right)^{2}=\left|\log \left(\frac{B}{A} \frac{\alpha}{\beta}\right)\right|^{2},
\end{aligned}
$$

where $\log$ is now the principal branch. We point out that (66) is clearly true when $\theta=\pi$. We will consider the cases $0<\theta<\pi$ and $\pi<\theta<2 \pi$. Using

$$
\frac{B}{A} \frac{\alpha}{\beta}=1+\frac{1-k}{1+k} \frac{\bar{\alpha}^{2}-\bar{\beta}^{2}}{1-\frac{1-k}{1+k} \bar{\alpha}^{2}}
$$

and the fact that for $k$ close to 1 these points are close to 1 , we find that

$$
\left|\log \left(\frac{B}{A} \frac{\alpha}{\beta}\right)\right|^{2} \leq\left(\frac{1-k}{k} \sin \theta\right)^{2} .
$$

For $0<\theta<\pi$ we apply (67) and bound the left hand side of (66) from below by

$$
|\sin \theta|\left(\left(4-\frac{2(1-k)}{k}\right) 2 \pi-4 \theta\right),
$$

and for $\pi<\theta<2 \pi$ we bound the left hand side of (66) from below by

$$
|\sin \theta|\left(4 \theta-4 \pi \frac{1-k}{k}\right) \text {. }
$$

Combining these two estimates with (67) will give (66) if

$$
4 \pi-4 \pi \frac{1-k}{k}>\left(\frac{1-k}{k}\right)^{2} \text {. }
$$

This may be solved using the quadratic formula, and we find that

$$
k>\frac{1}{2\left(\sqrt{\pi^{2}+\pi}-\pi\right)+1} \approx .518
$$

will work. This is a crude estimate, and the precise value of $k_{0}$ is probably much less than .518.

\section{REFERENCES}

[ADN64] S. Agmon, A. Douglis, and L. Nirenberg, Estimates near the boundary for solutions of elliptic partial differential equations satisfying general boundary conditions. ii., Comm. Pure Appl. Math 17 (1964), 35-92. MR 28:5252

[BG93] Heinrich Begehr and Robert P Gilbert, Transformations, transmutations, and kernel functions Volume 2, Pitman Monographs and Surveys in Pure and Applied Mathematics 59, Longman Scientific \& Technical, Copublished by John Wiley \& Sons Inc., New York, 1993. MR 95b:35001

[Bit48] A. V. Bitsadze, On the uniqueness of the solutions of the Dirichlet problem for elliptic partial differential equations, Uspehi Mat. Nauk 3 (1948), no. 6, 211-212. (Russian) MR 10:300f

[Cal77] A. P. Calderon, Cauchy integrals on Lipschitz curves and related operators, Proc. Nat. Acad. Sci. U.S.A. 74 (1977), 1324-1327. MR 57:6445

[CD93] M. Costabel and M. Dauge, Construction of corner singularities for Agmon-DouglisNirenberg elliptic systems, Math. Nachr. 162 (1993), 209-237. MR 94k:35090 
[CMM82] R. R. Coifman, A. McIntosh, and Y. Meyer, L'intégrale de Cauchy définit un opérateur borné sur $L^{2}$ pour les courbes Lipschitziennes, Ann. of Math. 116 (1982), 361-387. MR 84m:42027

[CW77] R. R. Coifman and G. Weiss, Extensions of Hardy spaces and their use in analysis, Bull. Amer. Math. Soc. 83 (1977), 569-646. MR 56:6264

[Dah77] B. E. J. Dahlberg, On estimates of harmonic measure, Arch. Rational Mech. Anal. 65 (1977), 272-288. MR 57:6470

[Dah79] B. E. J. Dahlberg, On the Poisson integral for Lipschitz and $C^{1}$ domains, Studia Math. 66 (1979), 13-24. MR 81g:31007

$\left[\mathrm{DK}^{+}\right] \quad$ B. E. J. Dahlberg, C. E. Kenig, J. Pipher, and G. C. Verchota, Area integral estimates and maximum principles for higher order elliptic equations and systems, in preparation.

[DK87] B. E. J. Dahlberg and C. E. Kenig, Hardy spaces and the Neumann problem in $L^{p}$ for Laplace's equation in Lipschitz domains, Annals of Mathematics 125 (1987), 437-465. MR 88d:35044

[DK90] B. E. J. Dahlberg and C. E. Kenig, $L^{p}$ estimates for the 3-dimensional systems of elastostatics on Lipschitz domains, Lecture Notes in Pure and Applied Math., vol. 122, Dekker, New York, 1990, pp. 621-634. MR 91h:35053

[DKV86] B. E. J. Dahlberg, C. E. Kenig, and G. C. Verchota, The Dirichlet problem for the biharmonic equation in a Lipschitz domain, Ann. Inst. Fourier(Grenoble) 36 (1986), 109-135. MR 88a:35070

[DKV88] B. E. J. Dahlberg, C. E. Kenig, and G. C. Verchota, Boundary value problems for the system of elastostatics in Lipschitz domains, Duke Math Journal 37 (1988), 795-818. MR 90d:35259

[DL92] L. Diomeda and B. Lisena, The Dirichlet problem for elliptic systems in piecewise $C^{1}$ plane domains, Indiana University Math Journal 41 (1992), no. 3, 649-670. MR 94a:35035

[FJL76] E. B. Fabes, M. Jodeit, and J. E. Lewis, On the spectra of a Hardy kernel, J. Funct. Anal. 21 (1976), 187-194. MR 52:15114

[FJL77] E. B. Fabes, M. Jodeit, and J. E. Lewis, Double layer potentials for domains with corners and edges, Indiana Univ. Math. J. 26 (1977), 95-114. MR 55:5879

[FJR78] E. B. Fabes, M. Jodeit, and N. M. Riviere, Potential techniques for boundary value problems on $C^{1}$ domains, Acta Math. 141 (1978), 165-186. MR 80b:31006

[FK81] E. B. Fabes and C. E. Kenig, On the Hardy space $H^{1}$ of a $C^{1}$ domain, Arkiv Mat. 19 (1981), 1-22. MR 84a:42029

[Gar81] John B. Garnett, Bounded analytic functions, Academic Press, New York, 1981. MR 83g:30037

[JK81] D. Jerison and C. E. Kenig, The Neumann problem on Lipschitz domains, Bull. Amer. Math. Soc. 4 (1981), 203-207. MR 84a:35064

[Joh55] Fritz John, Plane waves and spherical means applied to partial differential equations, Interscience, New York, 1955. MR 17:746d

[Ken80] C. E. Kenig, Weighted $H^{p}$ spaces on Lipschitz domains, American Journal of Mathematics 102 (1980), no. 1, 129-163. MR 81d:30060

[Koz90] V. A. Kozlov, On the singularities of solutions of the Dirichlet problem for elliptic equations in the neighborhood of corner points, Leningrad Math. J. 1 (1990), 967-982. MR 91c:35048

[KWCQ85] Hua Loo Keng, Lin Wei, and Wu Ci-Quian, Second-order systems of partial differential equations in the plane, Pitman Advanced Publishing Program, Boston London Melbourne, 1985. MR 88m:35002

[LP80] J. E. Lewis and C. Parenti, Pseudodifferential operators and Hardy kernels on $L^{p}\left(\mathbb{R}^{+}\right)$, Annali Sc. Norm. Sup. Pisa Ser IV 7 (1980), 481-503. MR 83d:47052

[LP83] J. E. Lewis and C. Parenti, Pseudodifferential operators of Mellin type, Comm. PDE 8 (1983), 477-544. MR 86f:35185

[PV93] J. Pipher and G. C. Verchota, The maximum principle for biharmonic functions in Lipschitz and $C^{1}$ domains, Commentarii Math. Helvetici 68 (1993), 385-414. MR 94j:35030

[PV95a] J. Pipher and G. C. Verchota, Dilation invariant estimates and the boundary Garding inequality for higher order elliptic operators, Ann. Of Math. 142 (1995), 1-38. MR 96g:35052 
[PV95b] J. Pipher and G. C. Verchota, Maximum principles for the polyharmonic equation on Lipschitz domains, Potential Analysis 4 (1995), 615-636. MR 96i:35021

$\left[\mathrm{SK}^{+} 60\right] \quad$ Ding Shia-Kuai et al., On the definition of the second order elliptic system of partial differential equations with constant coefficients, Acta Math. Sinica 10 (1960), 276287; English translation, Chinese Math. 1 (1960), 288-299. MR 27:2713

[Var77] N. T. Varopoulos, BMO functions and the $\bar{\partial}$-equation, Pacific J. Math. 71 (1977), 221-273. MR 58:22639a

[Ver84] G. C. Verchota, Layer potentials and regularity for the Dirichlet problem for Laplace's equation in Lipschitz domains, J. Funct. Anal. 59 (1984), no. 3, 572-611. MR 86e:35038

[Ver87] G. C. Verchota, The Dirichlet problem for the biharmonic equation in $C^{1}$ domains, Ind. Univ. Math J. 36 (1987), 867-895. MR 88m:35051

[Ver96] G. C. Verchota, Potentials for the Dirichlet problem in Lipschitz domains, Potential Theory-ICPT 94 (Kouty, 1994), de Gruyter, Berlin, 1996, pp. 167-187. CMP 96:17

Department of Mathematics, Syracuse University, Syracuse, New York 13244

E-mail address: alvogel@izebug.syr.edu 